\title{
14-Electron Four-Coordinate Ru(II) Carbyl Complexes and Their Five-Coordinate Precursors: Synthesis, Double Agostic Interactions, and Reactivity
}

\author{
Dejian Huang, ${ }^{\dagger}$ William E. Streib, ${ }^{\dagger}$ John C. Bollinger, ${ }^{\dagger}$ Kenneth G. Caulton, $*, \dagger$ \\ Rainer F. Winter, $*,+$ and Thomas Scheiring \\ Contribution from the Department of Chemistry and Molecular Structure Center, Indiana University, \\ Bloomington, Indiana 47405-4001, and Institut für Anorganische Chemie der Universität, \\ Pfaffenwaldring 55, D-70569 Stuttgart, Germany
}

Received February 26, 1999

\begin{abstract}
The structure of five-coordinate $\mathrm{Ru}(\mathrm{II})$ complexes $\mathrm{RuHCl}(\mathrm{CO})\left(\mathrm{P}^{i} \mathrm{Pr}_{3}\right)_{2}, \mathbf{1}, \mathrm{RuCl}_{2}(\mathrm{CO})\left(\mathrm{P}^{i} \mathrm{Pr}_{3}\right)_{2}, \mathbf{2}$, and $\mathrm{Ru}(\mathrm{Ph}) \mathrm{Cl}(\mathrm{CO})\left(\mathrm{P}^{t} \mathrm{Bu}_{2} \mathrm{Me}\right)_{2}, \mathbf{1 2}$, are reported. All three of these complexes have square-based pyramid geometry with the strongest $\sigma$-donor ligand trans to the vacant site. These 16-electron complexes do not show bona fide agostic interactions. This is attributed to the strong trans influence ligand $(\mathrm{H}, \mathrm{CO}$, and $\mathrm{Ph})$ and $\pi$-donation of the $\mathrm{Cl}$, which is further supported by the fact that two agostic interactions are present in the $\mathrm{Cl}^{-}$removal product of 12, i.e., the four-coordinate $\left[\mathrm{RuPh}(\mathrm{CO}) \mathrm{L}_{2}\right] \mathrm{BAr}{ }_{4}^{\prime}\left(\mathrm{L}=\mathrm{P}^{\prime} \mathrm{Bu}_{2} \mathrm{Me}, \mathrm{Ar}^{\prime}=3,5-\mathrm{C}_{6} \mathrm{H}_{3}\left(\mathrm{CF}_{3}\right)_{2}\right)$, 16. Structural comparison of $\mathbf{1 6}$ and $\mathbf{1 2}$ reveals that removal of $\mathrm{Cl}^{-}$does not change the remaining ligand arrangements but creates two low-lying LUMOs for agostic interactions, which persist in solution as evidenced by IR spectroscopy. Reactions of 16 with $\mathrm{E}-\mathrm{H}(\mathrm{E}=\mathrm{B}, \mathrm{C}(\mathrm{sp}))$ bonds cleave the $\mathrm{Ru}-\mathrm{Ph}$ bond and form $\mathrm{Ru}-\mathrm{E} / \mathrm{H}$ bonds by different mechanisms. The reaction with catecholborane gives $\left[\mathrm{RuH}(\mathrm{CO}) \mathrm{L}_{2}\right] \mathrm{BAr}_{4}{ }_{4}$, which further reacts with catecholborane to give $\left[\mathrm{Ru}\left(\mathrm{BR}_{2}\right)(\mathrm{CO}) \mathrm{L}_{2}\right] \mathrm{BAr}_{4}{ }_{4}$. However, the reaction with $\mathrm{Me}_{3} \mathrm{SiCCH}$ undergoes a multistep transformation to give a $\mathrm{PhCCSiMe}_{3}$ and $\mathrm{Me}_{3} \mathrm{SiCCH}$-coupled product, the mechanism of which is discussed. Reaction of $\mathrm{RuCl}_{2}(\mathrm{CO}) \mathrm{L}_{2}$ with 1 equiv MeLi affords $\mathrm{RuMeCl}(\mathrm{CO}) \mathrm{L}_{2}, \mathbf{5}$, which further reacts with MeLi forming $\mathrm{RuMe}_{2}(\mathrm{CO}) \mathrm{L}_{2}$, 7. Variable-temperature ${ }^{13} \mathrm{C}\left\{{ }^{1} \mathrm{H}\right\}$ NMR spectra reveal the two methyls in 7 are inequivalent and exchange by overcoming an energy barrier of $6.8 \mathrm{kcal} / \mathrm{mol}$ at $-30{ }^{\circ} \mathrm{C}$. The chloride of 5 can be removed to give $\left[\mathrm{RuMe}(\mathrm{CO}) \mathrm{L}_{2}\right] \mathrm{BAr}^{\prime}{ }_{4}$.
\end{abstract}

\section{Introduction}

Coordinatively and electronically unsaturated transition metal carbyl complexes are key species in promoted reactions such as olefin polymerization, hydrogenation, hydrosilylation and hydroboration. ${ }^{1}$ Particularly, complexes with formally 14valence electrons, or 16-electron but bearing an extremely labile ligand (e.g., agostic bonding, weakly coordinating counterion or solvent) are recognized as the active catalytic component of olefin polymerization reactions and are extensively studied, both on early and late transition metals (III-IVB, Ni, Pd, Pt). ${ }^{2}$ In sharp contrast, isolable 14-electron complexes of other transition metals are rare, although 14-electron species have been proposed often as active species in organometallic reactions. ${ }^{3}$ It has been proposed that a 14-electron complex has the advantage over its 16-electron counterpart since it provides two low-lying empty orbitals for substrate binding, and group (or atom) migration and bond formation. ${ }^{4} \mathrm{We}$ wish to report our results on the synthesis and structure of four-coordinate $\mathrm{Ru}(\mathrm{II})$ carbyl com-

* Corresponding author. E-mail: caulton@indiana.edu.

(1) Hegedus, L. S. In Comprehensive Organometallic Chemistry II; Abel, E. W., Stone, F. G. A., Wilkinson, G., Eds.; Pergamon: New York, 1995; p 1.

(2) (a) Grubbs, R. H.; Coates, G. W. Acc. Chem. Res. 1996, 29, 85. (b) Brintzinger, H. H.; Fischer, D.; Mulhaupt, R.; Rieger, B.; Waymouth, R. M. Angew. Chem., Int. Ed. Engl. 1995, 34, 1143 and references therein. (c) Johnson, L. K.; Kilian, C. M.; Brookhart, M. J. Am. Chem. Soc. 1995, 117, 6414.

(3) (a) Wick, D. D.; Goldberg, K. I. J. Am. Chem. Soc. 1997, 119, 10235 (b) Cooper, A. C.; Caulton, K. G. Inorg. Chim. Acta 1996, 251, 41. plexes with a 14-electron count, $\left[\mathrm{Ru}(\mathrm{R})(\mathrm{CO}) \mathrm{L}_{2}\right]^{+}\left(\mathrm{R}=\mathrm{CH}_{3}\right.$, $\mathrm{Ph}$, catecholboryl). ${ }^{5}$ The unusual structural feature of these complexes is the presence of two agostic interactions.

The geometry preference of five-coordinate $\mathrm{d}^{6}$ metal complexes has been well studied. ${ }^{6}$ The diamagnetic $\mathrm{Ru}$ or Os complexes with the general formula, $\mathrm{MXY}(\mathrm{CO}) \mathrm{L}_{2}(\mathrm{X}$ and $\mathrm{Y}$ are univalent ligands, $\mathrm{L}$ is usually a phosphine) adopt a squarebased pyramidal geometry with the strongest trans influence ligand at the apical site so that the LUMO has the highest possible energy. When the $\mathrm{X}$ and $\mathrm{Y}$ are significantly different (e.g., $\mathrm{H}$ vs $\mathrm{Cl}$ ) the geometry of the complex is rather obvious, but when the $\mathrm{X}$ and $\mathrm{Y}$ have similar trans influence (e.g., Me vs $\mathrm{H}$ or $\mathrm{Ph}$ ), predicting the geometry is not straightforward. Although these complexes are fluxional, the primary reaction product is usually governed by the ground-state geometry. ${ }^{7}$ On the route to 14-electron $\mathrm{Ru}$ alkyl complexes, we have synthesized several five-coordinate precursors where $\mathrm{X}$ and $\mathrm{Y}$ are carbyls of similar trans influence. Their geometry preferences

(4) Crabtree, R. H. In Activation and Functionalization of Methane; Davies, J. A., Ed.; VCH: New York, 1990; p 69.

(5) A preliminary communication has appeared: Huang, D.; Streib, W. E.; Eisenstein, O.; Caulton, K. G. Angew. Chem., Int. Ed. Engl. 1997, 36, 2004.

(6) (a) Cundari, T. R. J. Am. Chem. Soc. 1994, 116, 340. (b) Su, M.-D.; Chu, S.-Y. J. Am. Chem. Soc. 1997, 119, 10178.

(7) (a) Huang, D.; Spivak, G. J.; Caulton, K. G. New. J. Chem. 1998 22, 1023. (b) Huang, D.; Caulton, K. G. Inorg. Chem. 1996, 35, 7035. (c) Werner, H.; Stüer, W.; Laubender, M.; Lehmann, C.; Herbst-Irmer, R. Organometallics 1997, 16, 2236. 
Table 1. Crystallographic Data ${ }^{a}$

\begin{tabular}{|c|c|c|c|c|}
\hline & 1 & 2 & 12 & 16 \\
\hline formula & $\mathrm{C}_{19} \mathrm{H}_{43} \mathrm{ClOP}_{2} \mathrm{Ru}$ & $\mathrm{C}_{19} \mathrm{H}_{42} \mathrm{Cl}_{2} \mathrm{OP}_{2} \mathrm{Ru}$ & $\mathrm{C}_{25} \mathrm{H}_{47} \mathrm{ClOP}_{2} \mathrm{Ru}$ & $\mathrm{C}_{57} \mathrm{H}_{59} \mathrm{BF}_{24} \mathrm{OP}_{2} \mathrm{Ru}$ \\
\hline FW & 485.99 & 520.44 & 562.12 & 1389.89 \\
\hline color & orange & red & orange & thermochromic \\
\hline space group & $P 2_{1} / c$ & $C c$ & $P \overline{1}$ & $P 2{ }_{1} 2_{1}{ }_{1}$ \\
\hline$T(\mathrm{~K})$ & $173(2)$ & $183(2)$ & 103 & 103 \\
\hline$a(\AA)$ & $8.0675(5)$ & $21.867(4)$ & $16.680(3)$ & $18.176(3)$ \\
\hline$b(\AA)$ & $8.9312(7)$ & $8.648(2)$ & $17.037(4)$ & $18.495(3)$ \\
\hline$c(\AA)$ & $16.6316(10)$ & $15.033(2)$ & $10.772(2)$ & $18.090(3)$ \\
\hline$\alpha(\mathrm{deg})$ & 90 & 90 & $90.81(1)$ & 90 \\
\hline$\beta(\mathrm{deg})$ & $92.492(5)$ & $119.75(1)$ & $92.85(1)$ & 90 \\
\hline$\gamma(\mathrm{deg})$ & 90 & 90 & $112.68(1)$ & 90 \\
\hline$Z$ & 2 & 4 & 4 & 4 \\
\hline$V\left(\AA^{3}\right)$ & $1197.2(1)$ & $2468.1(7)$ & 2819.10 & $6081(46)$ \\
\hline$\rho_{\text {calc }}\left(\mathrm{g} / \mathrm{cm}^{3}\right)$ & 1.348 & 1.401 & 1.324 & 1.518 \\
\hline$\lambda(\AA)$ & 0.71073 & 0.71073 & 0.71069 & 0.71069 \\
\hline$\mu\left(\mathrm{cm}^{-1}\right)$ & 6.85 & 9.88 & 7.66 & 4.23 \\
\hline $\mathrm{wR}_{2}$ & 0.069 & 0.0727 & 0.0464 & 0.062 \\
\hline$R_{1}$ & 0.0264 & 0.0265 & 0.0655 & 0.061 \\
\hline
\end{tabular}

${ }^{a} R=\sum|| F_{\mathrm{o}}|-| F_{\mathrm{c}}|| / \sum\left|F_{\mathrm{o}}\right| ; R_{\mathrm{w}}=\left[\sum w\left(\left|F_{\mathrm{o}}\right|-\left|F_{\mathrm{c}}\right|\right)^{2} / \sum w\left|F_{\mathrm{o}}\right|^{2}\right]^{1 / 2}$, where $w=1 / \sigma^{2}\left(\left|F_{\mathrm{o}}\right|\right)$.

Table 2. Geometric Parameters of $\operatorname{RuHCl}(\mathrm{CO})\left(\mathrm{P}^{i} \mathrm{Pr}_{3}\right)_{2}$

\begin{tabular}{lccc}
\hline \multicolumn{4}{c}{ Bond Lengths $(\AA)$} \\
$\mathrm{Ru}-\mathrm{C}(3)$ & $1.752(6)$ & $\mathrm{Ru}-\mathrm{P}(1)$ & $2.3794(4)$ \\
$\mathrm{Ru}-\mathrm{Cl}(2)$ & $2.4219(18)$ & $\mathrm{C}(3)-\mathrm{O}(3)$ & $1.164(6)$ \\
& \multicolumn{4}{c}{ Bond Angles (deg) } \\
$\mathrm{P}(1)-\mathrm{Ru}-\mathrm{P}(1)^{\prime}$ & 180 & $\mathrm{C}(3)-\mathrm{Ru}-\mathrm{Cl}(2)$ & $177.3(2)$ \\
$\mathrm{P}(1)-\mathrm{Ru}-\mathrm{Cl}(2)$ & $89.34(5)$ & $\mathrm{C}(3)-\mathrm{Ru}(1)-\mathrm{P}(1)$ & $90.04(14)$ \\
$\mathrm{C}(12)-\mathrm{P}(1)-\mathrm{Ru}$ & $112.16(6)$ & $\mathrm{C}(13)-\mathrm{P}(1)-\mathrm{Ru}$ & $113.62(7)$ \\
$\mathrm{C}(11)-\mathrm{P}(1)-\mathrm{Ru}$ & $113.25(6)$ & $\mathrm{Ru}(1)-\mathrm{C}(3)-\mathrm{O}(3)$ & $178.6(8)$ \\
\hline
\end{tabular}

are discussed based on spectroscopic data and X-ray structural data of related complexes.

\section{Experimental Section}

General Procedures. All reactions and manipulations were conducted using standard Schlenk and argon-filled glovebox techniques. Solvents were dried according to routine methods, distilled under argon, and stored in airtight solvent bulbs with Teflon closures. The solvents were also freshly degassed by freeze-pump-thaw cycles before use. All NMR solvents were dried, vacuum-transferred, and stored in an argon-filled glovebox. ${ }^{1} \mathrm{H},{ }^{31} \mathrm{P},{ }^{19} \mathrm{~F}$, and ${ }^{13} \mathrm{C}$ NMR spectra were recorded on a Varian Gem XL300 or Unity I400 spectrometer. Chemical shifts are referenced to solvent peaks $\left({ }^{1} \mathrm{H},{ }^{13} \mathrm{C}\right)$, or external $\mathrm{H}_{3} \mathrm{PO}_{4}\left({ }^{31} \mathrm{P}\right)$ and $\mathrm{CFCl}_{3}\left({ }^{19} \mathrm{~F}\right)$. Infrared spectra were recorded on a Nicolet 510P FT-IR spectrometer. Elemental analyses were conducted on the Perkin-Elmer 2400 CHNS/O analyzer at the Department of Chemistry, Indiana University. $\mathrm{RuHCl}(\mathrm{CO}) \mathrm{L}_{2}{ }^{8}$ and $\mathrm{NaBAr}_{4}{ }_{4}{ }^{9}$ are prepared following literature procedures. Other chemicals are commercially available and degassed before use.

X-ray Crystal Structure Determination of $\operatorname{RuHCl}(\mathrm{CO})\left(\operatorname{PiPr}_{3}\right)_{2}$, 1 (Tables1 and 2). Orange crystals were obtained by slowly cooling a hot concentrated solution in a 3:1 MeOH/toluene mixture; Siemens four-circle diffractometer P4. Refinement used 209 parameters without constraints. Minimum and maximum peak of residual electron density

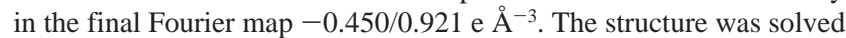
by direct methods (SHELXTL-Plus) and refined on $F^{2}$ (SHELXL93). Due to the special position of the ruthenium atom on a crystallographic inversion center, the $\mathrm{CO}$ and $\mathrm{Cl}$ ligand are disordered. The $\mathrm{C}(3), \mathrm{O}(3)$, and $\mathrm{Cl}(2)$ atoms were refined as "half occupied" (50\%). All non-H atoms were refined with anisotropic thermal parameters. All hydrogen atoms were introduced at their geometric positions and treated according to the "riding model" with isotopic thermal parameters fixed at $20 \%$ greater than that of the bonded $\mathrm{C}-\mathrm{H}$ atom.

$\left.\mathbf{R u C l}_{\mathbf{2}} \mathbf{C O}\right)\left(\mathbf{P}^{i} \mathbf{P r}_{3}\right)_{2}$, 2. RuHCl$(\mathrm{CO})\left(\mathrm{P}^{i} \mathrm{Pr}_{3}\right)_{2}(200 \mathrm{mg}, 0.41 \mathrm{mmol})$, $\mathrm{PhCH}_{2} \mathrm{Cl}(1.0 \mathrm{~g}, 8.2 \mathrm{mmol})$, and $10 \mathrm{~mL}$ of toluene were mixed in a

(8) Gill, D. F.; Shaw, B. L. Inorg. Chim. Acta 1979, 32, 19. Huang, D.; Folting, K.; Caulton, K. G. Inorg. Chem. 1996, 35, 7035.

(9) Brookhart, M.; Grant, B.; Volpe, J. Organometallics 1992, 11, 3920.
Table 3. Geometric Parameters of $\mathrm{RuCl}_{2}\left((\mathrm{CO})\left(\mathrm{P}^{i} \mathrm{Pr}_{3}\right)_{2}\right.$

\begin{tabular}{lccc}
\hline \multicolumn{4}{c}{ Bond Lengths $(\AA)$} \\
$\mathrm{Ru}-\mathrm{C} 1$ & $1.774(4)$ & $\mathrm{Ru}-\mathrm{Cl} 1$ & $2.358(2)$ \\
$\mathrm{Ru}-\mathrm{P} 2$ & $2.402(2)$ & $\mathrm{Ru}-\mathrm{P} 1$ & $2.406(3)$ \\
$\mathrm{C} 1-\mathrm{O} 1$ & $1.164(5)$ & $\mathrm{Ru}-\mathrm{Cl} 2$ & $2.382(3)$ \\
\multicolumn{4}{c}{ Bond Angles $(\mathrm{deg})$} \\
$\mathrm{C} 1-\mathrm{Ru}-\mathrm{Cl1}$ & $98.0(5)$ & $\mathrm{Cl} 1-\mathrm{Ru}-\mathrm{P} 1$ & $90.99(9)$ \\
$\mathrm{C} 1-\mathrm{Ru}-\mathrm{P} 2$ & $97.7(4)$ & $\mathrm{C} 15-\mathrm{P} 1-\mathrm{Ru}$ & $114.6(3)$ \\
$\mathrm{C} 1-\mathrm{Ru}-\mathrm{P} 1$ & $92.1(4)$ & $\mathrm{C} 25-\mathrm{P} 2-\mathrm{Ru}$ & $116.8(3)$ \\
$\mathrm{P} 2-\mathrm{Ru}-\mathrm{P} 1$ & $170.15(2)$ & $\mathrm{Cl1}-\mathrm{Ru}-\mathrm{Cl} 2$ & $165.97(3)$ \\
$\mathrm{C} 18-\mathrm{P} 1-\mathrm{Ru}$ & $114.7(3)$ & $\mathrm{Cl} 2-\mathrm{Ru}-\mathrm{P} 2$ & $90.89(9)$ \\
$\mathrm{C} 28-\mathrm{P} 2-\mathrm{Ru}$ & $112.1(3)$ & $\mathrm{C} 2-\mathrm{Ru}-\mathrm{P} 1$ & $87.66(9)$ \\
$\mathrm{C} 1-\mathrm{Ru}-\mathrm{Cl} 2$ & $96.0(5)$ & $\mathrm{C} 12-\mathrm{P} 1-\mathrm{Ru}$ & $110.2(3)$ \\
$\mathrm{C} 11-\mathrm{Ru}-\mathrm{P} 2$ & $88.06(9)$ & $\mathrm{C} 33-\mathrm{P} 2-\mathrm{Ru}$ & $106.7(3)$ \\
\hline
\end{tabular}

flask and heated at $80{ }^{\circ} \mathrm{C}$ for $4 \mathrm{~h}$. The solution color changed from orange to brown. The volatiles were evaporated in vacuo, and the residue were extracted with diethyl ether. The diethyl ether solution was evaporated to a give brown solid, which was recrystallized from toluene at $-40{ }^{\circ} \mathrm{C}$. Yield: $150 \mathrm{mg}(70 \%)$. Anal. Calcd for $\mathrm{C}_{19} \mathrm{H}_{42} \mathrm{Cl}_{2-}$ $\mathrm{OP}_{2} \mathrm{Ru}: \mathrm{C}, 43.84, \mathrm{H}, 8.07$. Found: C, 44.29, H, 7.78. ${ }^{1} \mathrm{H}$ NMR $(300$ $\left.\mathrm{MHz}, \mathrm{C}_{7} \mathrm{D}_{8}, 20^{\circ} \mathrm{C}\right): \delta 2.80\left(\mathrm{~m}, 6 \mathrm{H}, \mathrm{PCH}_{3}\right) 1.25\left(\mathrm{vdt}, J_{\mathrm{HH}}=6.5 \mathrm{~Hz}\right.$, $\left.N=14.4 \mathrm{~Hz}, 36 \mathrm{H}, \mathrm{PCH}\left(\mathrm{CH}_{3}\right)_{2}\right) .{ }^{31} \mathrm{P}\left\{{ }^{1} \mathrm{H}\right\} \mathrm{NMR}\left(145 \mathrm{MHz}, \mathrm{C}_{7} \mathrm{D}_{8}, 20\right.$ $\left.{ }^{\circ} \mathrm{C}\right): 44.8(\mathrm{~s}) . \operatorname{IR}\left(\mathrm{C}_{7} \mathrm{D}_{8}, \mathrm{~cm}^{-1}\right)$ : $1937(v(\mathrm{CO}))$.

$\mathrm{X}$-ray Crystal Structure Determination of $\mathrm{RuCl}_{2}(\mathrm{CO})\left(\mathrm{P}^{i} \mathbf{P r}_{3}\right)_{2}$. Single crystals were obtained by slow cooling of a hot saturated methanol solution of the compound. The single crystals were taken from the mother liquors, separated under Nujol, and sealed in a glass capillary. The data collection was performed on a Siemens-P4 fourcircle diffractometer. The structure was solved by the Patterson method, using the SHELXTL-Plus package. The refinement was carried out with SHELXL-93, employing full-matrix least-squares methods. Anisotropic thermal parameters were refined for all non-hydrogen atoms. All hydrogen atoms were constrained using a riding model with isotropic thermal parameters fixed at $20 \%$ greater than that of the bonded atom. The structure was refined (227 parameters) on $F^{2}$ (SHELXL93); the maximum and the minimum peaks in the final difference Fourier map corresponded to $-0.345 / 0.839 \mathrm{e} / \AA^{3}$ (Tables1 and 3).

$\mathbf{R u}\left(\mathrm{CH}_{3}\right) \mathbf{C l}(\mathbf{C O})\left(\mathbf{P}^{i} \mathbf{P r}_{3}\right)_{2}$, 4. $\mathrm{RuCl}_{2}(\mathrm{CO})\left(\mathrm{P}^{\mathrm{i}} \mathrm{Pr}_{3}\right)_{2}(150 \mathrm{mg}, 0.3 \mathrm{mmol})$ was dissolved in benzene $(5 \mathrm{~mL})$. To the solution, $\mathrm{MeLi}(1.4 \mathrm{~mol} / \mathrm{L}$ in diethyl ether, $0.21 \mathrm{~mL}$ ) was added, and the solution was stirred for 5 $\mathrm{h}$. The solvent was removed, and the residue was extracted with pentane and filtered. The filtrate was concentrated to $3 \mathrm{~mL}$ and cooled to -40 ${ }^{\circ} \mathrm{C}$ for 1 day to give orange crystals. ${ }^{1} \mathrm{H}$ NMR $\left(\mathrm{C}_{6} \mathrm{D}_{6}, 20{ }^{\circ} \mathrm{C}\right): 2.60(\mathrm{~m}$, $\left.6 \mathrm{H}, \mathrm{PCH}\left(\mathrm{CH}_{3}\right)_{2}\right), 1.52\left(\mathrm{t}, J=5 \mathrm{~Hz}, 3 \mathrm{H}, \mathrm{Ru}-\mathrm{CH}_{3}\right), 1.25(\mathrm{vtd}, N=$ $\left.13.5 \mathrm{~Hz}, J_{\mathrm{HH}}=7 \mathrm{~Hz}, 18 \mathrm{H}, \mathrm{PCH}\left(\mathrm{CH}_{3}\right)_{2}\right), 1.20(\mathrm{vtd}, N=13.5 \mathrm{~Hz}, J=$ $\left.7 \mathrm{~Hz}, 18 \mathrm{H}, \mathrm{PCH}\left(\mathrm{CH}_{3}\right)_{2}\right) .{ }^{31} \mathrm{P}\left\{{ }^{1} \mathrm{H}\right\} \mathrm{NMR}: 37.7$ (s).

NMR Study of the Reaction of $\mathrm{RuCl}_{2}(\mathrm{CO})\left(\mathrm{P}^{i} \mathrm{Pr}_{3}\right)_{2}$ with $\mathrm{MeLi}$. $\mathrm{RuCl}_{2}(\mathrm{CO})\left(\mathrm{P}^{i} \mathrm{Pr}_{3}\right)_{2}(10 \mathrm{mg}, 0.019 \mathrm{mmol})$ was dissolved in $\mathrm{C}_{6} \mathrm{H}_{6}(0.5$ 
$\mathrm{mL})$. To the solution, $\mathrm{MeLi}(1.4 \mathrm{~mol} / \mathrm{L}$ in diethyl ether, $14 \mu \mathrm{L})$ was added. After $10 \mathrm{~min}$, the ${ }^{31} \mathrm{P}\left\{{ }^{1} \mathrm{H}\right\}$ NMR spectrum reveals two products, $\mathrm{Ru}\left(\mathrm{CH}_{3}\right) \mathrm{Cl}(\mathrm{CO})\left(\mathrm{P}^{i} \mathrm{Pr}_{3}\right)_{2}$ and $\mathrm{Ru}\left(\mathrm{CH}_{3}\right)_{2}(\mathrm{CO})\left(\mathrm{P}^{i} \mathrm{Pr}_{3}\right)_{2}$, in equal amounts, along with starting material. After $3 \mathrm{~h}$, the ${ }^{31} \mathrm{P}\left\{{ }^{1} \mathrm{H}\right\}$ NMR spectrum reveals $\mathrm{Ru}\left(\mathrm{CH}_{3}\right) \mathrm{Cl}(\mathrm{CO})\left(\mathrm{P}^{i} \mathrm{Pr}_{3}\right)_{2}$ as the dominant product. To the same NMR tube, one more equivalent MeLi was added. After $10 \mathrm{~min}, \mathrm{Ru}-$ $\left(\mathrm{CH}_{3}\right)_{2}(\mathrm{CO})\left(\mathrm{P}^{i} \mathrm{Pr}_{3}\right)_{2}$ is the only product based on the ${ }^{31} \mathrm{P}\left\{{ }^{1} \mathrm{H}\right\} \mathrm{NMR}$ spectrum. The solvent of the reaction was removed, and to the same NMR tube, $\mathrm{C}_{6} \mathrm{D}_{6}(0.5 \mathrm{~mL})$ was added. ${ }^{1} \mathrm{H}$ NMR $\left(300 \mathrm{MHz}, 20{ }^{\circ} \mathrm{C}\right)$ : $2.51\left(\mathrm{~m}, \mathrm{PCH}\left(\mathrm{CH}_{3}\right)_{2}\right), 1.12(\mathrm{dvt}, J=6 \mathrm{~Hz}, N=12.4 \mathrm{~Hz}, 36 \mathrm{H}, \mathrm{PCH}-$ $\left.\left(\mathrm{CH}_{3}\right)_{2}\right), 0.82\left(\mathrm{t}, J=6 \mathrm{~Hz}, 6 \mathrm{H}, \mathrm{Ru}-\mathrm{CH}_{3}\right) .{ }^{31} \mathrm{P}\left\{{ }^{1} \mathrm{H}\right\} \mathrm{NMR}: 40.7$ (s). IR $\left(\mathrm{C}_{6} \mathrm{D}_{6}\right): 1894(v(\mathrm{CO}))$.

$\mathbf{R u}\left(\mathbf{C H}_{3}\right)_{2}(\mathbf{C O})\left(\mathbf{P}^{t} \mathbf{B u}_{2} \mathbf{M e}\right)_{2}$, 7. $\mathrm{RuCl}_{2}(\mathrm{CO})\left(\mathrm{P}^{t} \mathrm{Bu}_{2} \mathrm{Me}\right)_{2}(150 \mathrm{mg}, 0.29$ $\mathrm{mmol}$ ) was dissolved in benzene $(5 \mathrm{~mL})$. To the solution, MeLi (1.4 $\mathrm{mol} / \mathrm{L}$ in diethyl ether, $0.42 \mathrm{~mL}$ ) was added and stirred for $5 \mathrm{~min}$. The solvent was removed and residue was extracted with cold tetramethylsilane and filtered through a Celite pad. The filtrate was concentrated to $1 \mathrm{~mL}$ and cooled to $-78{ }^{\circ} \mathrm{C}$ for $24 \mathrm{~h}$. Brown crystals were formed, filtered, and washed with tetramethylsilane. Yield: $66 \%$. ${ }^{1} \mathrm{H}$ NMR $(300$ $\mathrm{MHz}, \mathrm{C}_{7} \mathrm{D}_{8}, 20{ }^{\circ} \mathrm{C}$ ): 1.28 (vt, $\left.N=5.4 \mathrm{~Hz}, 6 \mathrm{H}, \mathrm{PCH}_{3}\right), 1.07$ (vt, $N=$ $\left.11.8 \mathrm{~Hz}, 36 \mathrm{H}, \mathrm{PC}\left(\mathrm{CH}_{3}\right)_{3}\right), 0.52\left(\mathrm{t}, J=6 \mathrm{~Hz}, \mathrm{Ru}-\mathrm{CH}_{3}\right) .{ }^{31} \mathrm{P}\left\{{ }^{1} \mathrm{H}\right\} \mathrm{NMR}$ $\left(121 \mathrm{MHz}, 20{ }^{\circ} \mathrm{C}\right): 43.3$ (s) ${ }^{13} \mathrm{C}\left\{{ }^{1} \mathrm{H}\right\} \mathrm{NMR}\left(100 \mathrm{MHz}, 20{ }^{\circ} \mathrm{C}, \mathrm{C}_{7} \mathrm{D}_{8}\right)$ : $201.9\left(\mathrm{t}, J_{\mathrm{PC}}=12 \mathrm{~Hz}, \mathrm{Ru}-\mathrm{CO}\right), 36.6\left(\mathrm{vt}, N=15 \mathrm{~Hz}, \mathrm{PC}\left(\mathrm{CH}_{3}\right)_{3}\right), 29.7$ $\left(\mathrm{s}, \mathrm{PC}\left(\mathrm{CH}_{3}\right)_{3}\right), 2.6\left(\mathrm{t}, J_{\mathrm{PC}}=7.1 \mathrm{~Hz}, \mathrm{Ru}-\mathrm{CH}_{3}\right), 1.10(\mathrm{vt}, N=5 \mathrm{~Hz}$, $\left.\mathrm{PCH}_{3}\right) .-90{ }^{\circ} \mathrm{C}: 202.5\left(\mathrm{t}, J_{\mathrm{PC}}=13 \mathrm{~Hz}, \mathrm{Ru}-\mathrm{CO}\right), 37.2(\mathrm{vt}, N=17$ $\left.\mathrm{Hz}, \mathrm{PC}\left(\mathrm{CH}_{3}\right)_{3}\right), 36.4\left(\mathrm{vt}, N=17 \mathrm{~Hz}, \mathrm{PC}\left(\mathrm{CH}_{3}\right)_{3}\right), 29.3\left(\mathrm{br}, v_{1 / 2}=56\right.$ $\left.\mathrm{Hz}, \mathrm{PC}\left(\mathrm{CH}_{3}\right)_{3}\right), 29.6\left(\mathrm{br}, \mathrm{PC}\left(\mathrm{CH}_{3}\right)_{3}\right), 17.5\left(\mathrm{t}, J=11 \mathrm{~Hz}, \mathrm{Ru}-\mathrm{CH}_{3}\right)$, $1.14\left(\mathrm{br}, \mathrm{P}-\mathrm{CH}_{3}\right),-15.7\left(\mathrm{t}, J=7.5 \mathrm{~Hz}, \mathrm{Ru}-\mathrm{CH}_{3}\right)$. IR $\left(\mathrm{C}_{6} \mathrm{D}_{6}\right): 1878$ $(v(\mathrm{CO}))$.

$\mathbf{R u}\left(\mathrm{CH}_{3}\right) \mathbf{F}(\mathbf{C O})\left(\mathbf{P}^{t} \mathrm{Bu}_{2} \mathrm{Me}\right)_{2}, \mathbf{8} . \mathrm{Ru}\left(\mathrm{CH}_{3}\right) \mathrm{Cl}(\mathrm{CO})\left(\mathrm{P}^{t} \mathrm{Bu}_{2} \mathrm{Me}\right)_{2}(0.50 \mathrm{~g}$, $1.0 \mathrm{mmol})$ and $\mathrm{CsF}(0.5 \mathrm{~g}, 3.3 \mathrm{mmol})$ was mixed with acetone $(20$ $\mathrm{mL}$ ) and stirred for $4 \mathrm{~h}$. The volatiles were then removed in vacuo, and the residue was extracted with pentane and filtered. The filtrate was concentrated to ca. $5 \mathrm{~mL}$ and cooled to $-40{ }^{\circ} \mathrm{C}$ for 2 days. Brown crystals were filtered and washed with pentane $\left(-78^{\circ} \mathrm{C}\right)$. Yield: 0.26 g (54\%). Anal. Calcd for $\mathrm{C}_{20} \mathrm{H}_{45} \mathrm{FOP}_{2} \mathrm{Ru}$ : C, 49.67, H, 9.38. Found: C, 49.96, H, 9.00. ${ }^{1} \mathrm{H}$ NMR $\left(400 \mathrm{MHz}, \mathrm{C}_{6} \mathrm{D}_{6}, 20{ }^{\circ} \mathrm{C}\right): 1.32$ (t, $J=5$ $\left.\mathrm{Hz}, \mathrm{Ru}-\mathrm{CH}_{3}\right), 1.30$ (vt, $\left.N=12.4 \mathrm{~Hz}, 18 \mathrm{H}, \mathrm{PC}\left(\mathrm{CH}_{3}\right)_{3}\right), 1.20$ (vt, $N=$ $\left.12.4 \mathrm{~Hz}, 18 \mathrm{H}, \mathrm{PC}\left(\mathrm{CH}_{3}\right)_{3}\right), 1.09$ (vt, $\left.N=5 \mathrm{~Hz}, \mathrm{P}-\mathrm{CH}_{3}\right) .{ }^{31} \mathrm{P}\left\{{ }^{1} \mathrm{H}\right\}-$ NMR: $41.0\left(\mathrm{~d}, J_{\mathrm{PF}}=22 \mathrm{~Hz}\right) \cdot{ }^{19} \mathrm{~F}$ NMR: $-201\left(\mathrm{tq}, J_{\mathrm{PF}}=22 \mathrm{~Hz}, J_{\mathrm{FH}}\right.$ $=4 \mathrm{~Hz}, \mathrm{Ru}-\mathrm{F})$. IR $\left(\mathrm{C}_{6} \mathrm{D}_{6}\right): 1887(v(\mathrm{CO}))$.

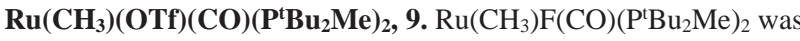
dissolved in diethyl ether $(10 \mathrm{~mL})$. To the solution, $\mathrm{Me}_{3} \operatorname{SiOTf}(34 \mu \mathrm{L})$ was added. The mixture was stirred for $10 \mathrm{~min}$ and filtered. The filtrate was concentrated to $2 \mathrm{~mL}$ and cooled to $-40{ }^{\circ} \mathrm{C}$ for 1 day to give yellow crystals. Yield: $90 \mathrm{mg}(70 \%)$. Anal. Calcd for $\mathrm{C}_{21} \mathrm{H}_{45} \mathrm{~F}_{3} \mathrm{O}_{4} \mathrm{P}_{2-}$ RuS: C, 41.10, H, 7.39. Found; C, 41.01, H, 7.43. ${ }^{1} \mathrm{H}$ NMR (300 MHz, $\mathrm{C}_{6} \mathrm{D}_{6}, 20{ }^{\circ} \mathrm{C}$ ): 1.49 (vt, $\left.\mathrm{N}=4 \mathrm{~Hz}, 6 \mathrm{H}, \mathrm{PCH}_{3}\right), 1.43$ (t, $J=5.2 \mathrm{~Hz}$, $\left.3 \mathrm{H}, \mathrm{RuCH}_{3}\right), 0.97$ (vt, $\left.N=13 \mathrm{~Hz}, \mathrm{PC}\left(\mathrm{CH}_{3}\right)_{3}\right), 0.94$ (vt, $N=13 \mathrm{~Hz}$, $\left.\mathrm{PC}\left(\mathrm{CH}_{3}\right)_{3}\right) \cdot{ }^{31} \mathrm{P}\left\{{ }^{1} \mathrm{H}\right\}$ NMR $\left(121 \mathrm{MHz}, 20{ }^{\circ} \mathrm{C}\right): 42.4$ (s). ${ }^{19} \mathrm{~F}$ NMR $(282$ $\left.\mathrm{MHz}, 20^{\circ} \mathrm{C}\right):-81.0\left(\mathrm{~s}, \mathrm{CF}_{3} \mathrm{SO}_{3}\right)$. IR $\left(\mathrm{C}_{6} \mathrm{D}_{6}\right): 1914(v(\mathrm{CO}))$.

$\mathbf{R u}\left(\mathbf{C H}_{3}\right)\left(\mathbf{B F}_{4}\right)(\mathbf{C O})\left(\mathbf{P}^{t} \mathbf{B u}_{2} \mathrm{Me}\right)_{2}, \mathbf{1 0} . \mathrm{Ru}\left(\mathrm{CH}_{3}\right) \mathrm{F}(\mathrm{CO})\left(\mathrm{P}^{\mathrm{t} B u_{2}} \mathrm{Me}\right)_{2}(50$ $\mathrm{mg}, 0.10 \mathrm{mmol})$ was dissolved in diethyl ether $(2 \mathrm{~mL})$. To the solution, $\mathrm{BF}_{3} \cdot \mathrm{OEt}_{2}(13 \mu \mathrm{L}, 0.1 \mathrm{mmol})$ was added. The mixture was stirred for $1 \mathrm{~min}$ and kept undisturbed for $1 \mathrm{~h}$ to get orange microcrystals. The solution was cooled to $-78{ }^{\circ} \mathrm{C}$ for one more hour, to obtain more product. The solvent was removed and the crystals were washed with cold diethyl ether and dried. Yield: $35 \mathrm{mg}(63 \%)$. ${ }^{1} \mathrm{H}$ NMR $(300 \mathrm{MHz}$, $\left.\mathrm{C}_{6} \mathrm{D}_{6}, 20^{\circ} \mathrm{C}\right): 1.47\left(\mathrm{vt}, N=5.3 \mathrm{~Hz}, 6 \mathrm{H}, \mathrm{PCH}_{3}\right), 1.30$ (vt, $N=12.8 \mathrm{~Hz}$, $\left.18 \mathrm{H}, \mathrm{PC}\left(\mathrm{CH}_{3}\right)_{3}\right), 1.22$ (vt, $\left.N=12.8 \mathrm{~Hz}, 18 \mathrm{H}, \mathrm{PC}\left(\mathrm{CH}_{3}\right)_{3}\right), \mathrm{Ru}-\mathrm{CH}_{3}$ protons are overlapping with ${ }^{\mathrm{t}} \mathrm{Bu}$ proton signals and not assigned. ${ }^{31} \mathrm{P}-$ $\left\{{ }^{1} \mathrm{H}\right\}$ NMR: $43.8(\mathrm{~s}),{ }^{19} \mathrm{~F}$ NMR: $-210\left(\mathrm{br}, v_{1 / 2}=1396 \mathrm{~Hz}, \mathrm{BF}_{4}\right)$. IR $\left(\mathrm{C}_{6} \mathrm{D}_{6}\right): 1919(v(\mathrm{CO}))$.

$\left[\mathbf{R u}\left(\mathbf{C H}_{3}\right)(\mathbf{C O})\left(\mathbf{P}^{\prime} \mathrm{Bu}_{2} \mathrm{Me}\right)_{2}\right] \mathrm{BAr}^{\prime}{ }_{4}$, 11. $\mathrm{Ru}\left(\mathrm{CH}_{3}\right)\left(\mathrm{BF}_{4}\right)(\mathrm{CO})\left(\mathrm{P}^{\prime} \mathrm{Bu}_{2^{-}}\right.$ $\mathrm{Me})_{2}(10 \mathrm{mg}, 0.018 \mathrm{mmol})$ and $\mathrm{NaBAr}_{4}{ }_{4}(16 \mathrm{mg}, 0.018 \mathrm{mmol})$ were mixed in $1: 2 \mathrm{C}_{6} \mathrm{D}_{5} \mathrm{~F} / \mathrm{C}_{7} \mathrm{D}_{8}$ mixture. The solution was stirred for $10 \mathrm{~min}$. Some colorless precipitate forms $\left(\mathrm{NaBF}_{4}\right) .{ }^{1} \mathrm{H}$ NMR $(400 \mathrm{MHz}, 20$ ${ }^{\circ} \mathrm{C}$ ): 8.28 (br, $8 \mathrm{H}$, ortho $\mathrm{H}$ of $\left.\mathrm{Ar}^{\prime}\right), 7.64$ (br, $4 \mathrm{H}$, para $\mathrm{H}$ of $\left.\mathrm{Ar}^{\prime}\right), 1.14$, (br, $3 \mathrm{H}, \mathrm{Ru}-\mathrm{CH}_{3}$ ), 1.07 (vt, $N=5 \mathrm{~Hz}, 6 \mathrm{H}, \mathrm{PCH}_{3}$ ), 0.91 (vt, $N=12.8$ $\left.\mathrm{Hz}, 18 \mathrm{H}, \mathrm{PC}\left(\mathrm{CH}_{3}\right)_{3}\right), 0.81$ (vt, $\left.N=12.8 \mathrm{~Hz}, 18 \mathrm{H}, \mathrm{PC}\left(\mathrm{CH}_{3}\right)_{3}\right) .{ }^{31} \mathrm{P}\left\{{ }^{1} \mathrm{H}\right\}$ NMR: 41.0 (s). ${ }^{19} \mathrm{~F}$ NMR: $-62\left(\mathrm{~s}, \mathrm{CF}_{3}\right.$ of $\left.\mathrm{Ar}^{\prime}\right)$. IR: $1951(v(\mathrm{CO}))$. At
Table 4. Selected Geometric Parameters of $\mathrm{RuPhCl}(\mathrm{CO})\left(\mathrm{P}^{\prime} \mathrm{Bu}_{2} \mathrm{Me}\right)_{2}$

\begin{tabular}{lcc}
\hline & molecule A & molecule B \\
\hline & Bond Lengths $(\AA)$ & \\
$\mathrm{Ru}(1)-\mathrm{Cl}(2)$ & $2.4432(6)$ & $2.4534(6)$ \\
$\mathrm{Ru}(1)-\mathrm{P}(3)$ & $2.4097(5)$ & $2.4178(5)$ \\
$\mathrm{Ru}(1)-\mathrm{P}(13)$ & $2.4296(5)$ & $2.4259(5)$ \\
$\mathrm{Ru}(1)-\mathrm{C}(23)$ & $2.0394(4)$ & $2.0438(4)$ \\
$\mathrm{Ru}(1)-\mathrm{C}(29)$ & $1.8236(4)$ & $1.8129(4)$ \\
$\mathrm{O}(30)-\mathrm{C}(29)$ & $1.0871(2)$ & $1.0736(2)$ \\
& & \\
$\mathrm{C} 12-\mathrm{Ru} 1-\mathrm{P} 3$ & Bond Angles (deg) & \\
$\mathrm{C} 12-\mathrm{Ru} 1-\mathrm{C} 23$ & $87.685(14)$ & $88.912(14)$ \\
$\mathrm{P} 3-\mathrm{Ru} 1-\mathrm{C} 29$ & $102.948(14)$ & $90.199(14)$ \\
$\mathrm{P} 13-\mathrm{Ru} 1-\mathrm{C} 29$ & $92.293(14)$ & $105.167(13)$ \\
$\mathrm{Ru} 1-\mathrm{P} 3-\mathrm{C} 9$ & $86.924(15)$ & $165.042(5)$ \\
$\mathrm{Ru} 1-\mathrm{P} 3-\mathrm{C} 4$ & $121.222(15)$ & $173.6980(20)$ \\
$\mathrm{Ru} 1-\mathrm{P} 13-\mathrm{C} 15$ & $114.408(13)$ & $93.210(18)$ \\
$\mathrm{C} 12-\mathrm{Ru} 1-\mathrm{P} 13$ & $110.270(17)$ & $90.804(14)$ \\
$\mathrm{C} 2-\mathrm{Ru} 1-\mathrm{C} 29$ & $91.457(14)$ & $93.044(18)$ \\
$\mathrm{P} 13-\mathrm{Ru} 1-\mathrm{C} 23$ & $167.143(4)$ & $88.446(14)$ \\
$\mathrm{C} 23-\mathrm{Ru} 1-\mathrm{C} 29$ & $92.736(18)$ & $89.782(15)$ \\
$\mathrm{Ru} 1-\mathrm{P} 3-\mathrm{C} 5$ & $89.874(15)$ & $126.188(11)$ \\
$\mathrm{Ru} 1-\mathrm{P} 13-\mathrm{C} 14$ & $105.760(17)$ & $117.328(15)$ \\
$\mathrm{Ru} 1-\mathrm{P} 13-\mathrm{C} 19$ & $112.079(13)$ & $179.8500(10)$ \\
\hline
\end{tabular}

$-70{ }^{\circ} \mathrm{C}$, the proton chemical shift of the methyl bound to Ru does not change much compared to that of $20^{\circ} \mathrm{C}$; therefore, no agostic interaction from the $\mathrm{Ru}-\mathrm{CH}_{3}$ is likely.

$\mathbf{R u}(\mathbf{P h}) \mathbf{C l}(\mathbf{C O})\left(\mathbf{P}^{t} \mathbf{B u}_{2} \mathbf{M e}\right)_{2}$, 12. (a) From $\mathbf{P h}_{2} \mathbf{H g}$. A toluene $(40 \mathrm{~mL})$ solution of $\mathrm{RuHCl}(\mathrm{CO})\left(\mathrm{P}^{t} \mathrm{Bu}_{2} \mathrm{Me}\right)_{2}(2.0 \mathrm{~g}, 4.1 \mathrm{mmol})$ and $\mathrm{Ph}_{2} \mathrm{Hg}(2.9$ $\mathrm{g}, 8.0 \mathrm{mmol}$ ) was refluxed for $12 \mathrm{~h}$ and freed of volatiles. ${ }^{10}$ The residue was extracted with pentane (ca. $120 \mathrm{~mL}$ ), which was evaporated to dryness to afford a crude product. Recrystallization from methanol gave dark-orange crystals. Yield: $1.92 \mathrm{~g}(83 \%) .{ }^{1} \mathrm{H}$ NMR $\left(\mathrm{C}_{7} \mathrm{D}_{8}, 20{ }^{\circ} \mathrm{C}\right)$ : $8.32(\mathrm{~d}, J=7.2 \mathrm{~Hz}, 1 \mathrm{H}$, ortho $\mathrm{H}$ of $\mathrm{Ph}), 7.31(\mathrm{~d}, J=7.2 \mathrm{~Hz}, 1 \mathrm{H}$, ortho $\mathrm{H}$ of $\mathrm{Ph}), 6.78(\mathrm{~m}, 1 \mathrm{H}$, para $\mathrm{H}$ of $\mathrm{Ph}), 6.69(\mathrm{~m}, 2 \mathrm{H}$, meta $\mathrm{H}$ of $\mathrm{Ph}), 1.44$ (vt, $N=5.4 \mathrm{~Hz}, 6 \mathrm{H}, \mathrm{PMe}), 1.04$ (vt, $\left.N=12 \mathrm{~Hz}, 18 \mathrm{H}, \mathrm{P}^{\prime} \mathrm{Bu}\right)$, 1.02(vt, $\left.N=12 \mathrm{~Hz}, 18 \mathrm{H}, \mathrm{P}^{\prime} \mathrm{Bu}\right) .{ }^{31} \mathrm{P}\left\{{ }^{1} \mathrm{H}\right\} \mathrm{NMR}\left(\mathrm{C}_{7} \mathrm{D}_{8}, 20{ }^{\circ} \mathrm{C}\right): 34.0$ (s). IR $\left(\mathrm{C}_{6} \mathrm{D}_{6}, \mathrm{~cm}^{-1}\right): v(\mathrm{CO})=1902$.

(b) From $\mathbf{R u C l}_{\mathbf{2}}(\mathbf{C O})\left(\mathbf{P}^{t} \mathbf{B u}_{2} \mathbf{M e}\right)_{2}$ and $\mathbf{P h L i}$. $\mathrm{RuCl}_{2}(\mathrm{CO}) \mathrm{L}_{2}(100 \mathrm{mg}$, $0.19 \mathrm{mmol})$ was dissolved in a 9:1 pentane/toluene mixture $(5 \mathrm{~mL})$ and cooled to $-78^{\circ} \mathrm{C}$. $\mathrm{PhLi}(1.8 \mathrm{M}$ in cyclohexane/ether solution, 160 $\mu \mathrm{L}, 0.29 \mathrm{mmol}$ ) was added to the mixture. The mixture was stirred and warmed slowly (over $12 \mathrm{~h}$ ) to room temperature. The volatiles were evaporated, and the residue was recrystallized from methanol to give orange crystals. Yield: $85 \mathrm{mg}$ (79\%).

$\mathrm{X}$-ray Crystal Structure Determination of $\mathrm{RuPhCl}(\mathrm{CO})\left(\mathrm{P}^{\mathrm{t}} \mathrm{Bu}_{2} \mathrm{Me}\right)_{2}$ (Tables1 and 4). A small, well-formed crystal was chosen from the bulk sample and affixed to the tip of a glass fiber with the use of silicone grease. The mounted sample was then transferred to the goniostat and cooled to $-164{ }^{\circ} \mathrm{C}$ for data collection. A systematic search of a limited hemisphere of reciprocal space located a set of data with no symmetry or systematic absences, thus indicating a triclinic space group. Subsequent solution and refinement of the structure confirmed the choice of the centrosymmetric space group. Data were collected by the moving crystal, moving detector technique with fixed background counts at each extreme of the scan. Data were corrected for Lorentz and polarization effects, and equivalent data were averaged. The structure was solved by direct methods (SHELXTL) and Fourier techniques. Hydrogen atoms were placed in calculated positions and not refined. The final difference electron density map was featureless, with the highest peak having an intensity of $1.38 \mathrm{e} / \AA^{3}$ and residing near one $\mathrm{Ru}$ atom. There was no detectable disorder, and a least-squares fit of the coordinates of one independent molecule to those of the other indicates that the two have a close mirror image relationship. Given that there are two chemically identical independent molecules in the triclinic unit cell, one might suspect that a phase change had occurred

(10) Rickard, C. E. F.; Roper, W. R.; Taylor, G. E.; Waters, J. M.; Wright, L. J. J. Organomet. Chem. 1990, 389, 375. 
as the crystal was cooled. A check of the unit cell parameters at -55 ${ }^{\circ} \mathrm{C}$ indicated no significant change from those measured at $-164{ }^{\circ} \mathrm{C}$ however, so any phase change that may have taken place occurred above $-55^{\circ} \mathrm{C}$.

$\mathbf{R u}(\mathbf{P h}) \mathbf{F}(\mathbf{C O})\left(\mathbf{P}^{t} \mathrm{Bu}_{2} \mathbf{M e}\right)_{2}$, 13. $\mathrm{Ru}(\mathrm{Ph}) \mathrm{Cl}(\mathrm{CO})\left(\mathrm{P}^{t} \mathrm{Bu}_{2} \mathrm{Me}\right)_{2}(200 \mathrm{mg}$, $0.36 \mathrm{mmol})$ and $\mathrm{CsF}(100 \mathrm{mg}, 0.66 \mathrm{mmol})$ were mixed with acetone $(5 \mathrm{~mL})$ and stirred for $12 \mathrm{~h}$. The mixture was filtered and the residue was washed with pentane. The combined filtrate was evaporated to dryness in vacuo. The crude product was recrystallized from pentane $\left(-40{ }^{\circ} \mathrm{C}\right)$. Yield: $150 \mathrm{mg}(77 \%) .{ }^{1} \mathrm{H}$ NMR $\left(300 \mathrm{MHz}, \mathrm{C}_{6} \mathrm{D}_{6}, 20{ }^{\circ} \mathrm{C}\right)$ : $8.44\left(\mathrm{~d}, J_{\mathrm{HH}}=6.6 \mathrm{~Hz}, 1 \mathrm{H}\right.$, ortho $\mathrm{H}$ of $\left.\mathrm{Ph}\right), 7.50\left(\mathrm{~d}, J_{\mathrm{HH}}=8.1 \mathrm{~Hz}, 1 \mathrm{H}\right.$, ortho $\mathrm{H}$ of $\mathrm{Ph}), 6.92(\mathrm{~m}, 1 \mathrm{H}$, para $\mathrm{H}$ of $\mathrm{Ph}), 6.90(\mathrm{~m}$, meta $\mathrm{H}$ of $\mathrm{Ph}$ ), $6.83(\mathrm{~m}$, meta $\mathrm{H}$ of $\mathrm{Ph}), 1.31$ (vt, $\left.N=5.7 \mathrm{~Hz}, 6 \mathrm{H}, \mathrm{PCH}_{3}\right), 1.08$ (vt, $N$ $\left.=13.2 \mathrm{~Hz}, 18 \mathrm{H}, \mathrm{P}^{t} \mathrm{Bu}\right), 0.99\left(\mathrm{vt}, N=12.6 \mathrm{~Hz}, 18 \mathrm{H}, \mathrm{P}^{t} \mathrm{Bu}\right) .{ }^{31} \mathrm{P}\left\{{ }^{1} \mathrm{H}\right\}$ NMR $\left(121 \mathrm{~Hz}, \mathrm{C}_{6} \mathrm{D}_{6}, 20{ }^{\circ} \mathrm{C}\right): 42.0\left(\mathrm{~d}, J_{\mathrm{PF}}=24 \mathrm{~Hz}, \mathrm{Ru}-\mathrm{P}\right) .{ }^{19} \mathrm{~F}$ NMR $\left(376 \mathrm{MHz}, \mathrm{C}_{6} \mathrm{D}_{6}, 20^{\circ} \mathrm{C}\right):-204.5(\mathrm{t}, J=24 \mathrm{~Hz}, \mathrm{Ru}-\mathrm{F})$. IR $\left(\mathrm{C}_{6} \mathrm{D}_{6}\right.$, $\left.\mathrm{cm}^{-1}\right): v(\mathrm{CO})=1890$.

$\operatorname{Ru}(\mathbf{P h}) O T f(C O)\left(P^{t} B_{2} u_{2} M\right)_{2}$, 14. (a) From $\mathrm{Ph}_{2} H g$. $\operatorname{RuH}(\mathrm{OTf})(\mathrm{CO})-$ $\left(\mathrm{P}^{t} \mathrm{Bu}_{2} \mathrm{Me}\right)_{2}(0.50 \mathrm{~g}, 0.83 \mathrm{mmol})$ and $\mathrm{Ph}_{2} \mathrm{Hg}(0.50 \mathrm{~g}, 1.4 \mathrm{mmol})$ were mixed in toluene $(10 \mathrm{~mL})$. The mixture was refluxed for $12 \mathrm{~h}$, during which time mercury metal precipitates. The solution was cooled to room temperature and filtered through a Celite pad. The filtrate was evaporated to dryness. The resulting orange solid was heated in vacuo at $80{ }^{\circ} \mathrm{C}$ to sublime away excess $\mathrm{Ph}_{2} \mathrm{Hg}$. The remaining orange solid was dissolved in diethyl ether and filtered. The filtrate was concentrated to $3 \mathrm{~mL}$ and layered with pentane. Orange crystals were formed over 1 week. Yield: $0.45 \mathrm{~g}(80 \%)$.

(b) From $\mathrm{Ru}(\mathrm{Ph}) \mathrm{F}(\mathrm{CO})\left(\mathrm{P}^{t} \mathrm{Bu}_{2} \mathrm{Me}\right)_{2}$ and $\mathrm{Me}_{3} \mathrm{SiOTf}$. RuPhF(CO)$\left(\mathrm{P}^{t} \mathrm{Bu}_{2} \mathrm{Me}\right)_{2}(150 \mathrm{mg}, 0.28 \mathrm{mmol})$ was dissolved in cyclohexane $(10$ $\mathrm{mL}$ ). To the solution, $\mathrm{Me}_{3} \operatorname{SiOTf}(54 \mu \mathrm{L}, 0.28 \mathrm{mmol}$ ) was added. The mixture was stirred for $10 \mathrm{~min}$ and freed of volatiles. Recrystallization from toluene layered with pentane gave orange crystals. Yield: 110 mg $(58 \%)$. ${ }^{1} \mathrm{H}$ NMR $\left(300 \mathrm{MHz}, \mathrm{C}_{6} \mathrm{D}_{6}, 20{ }^{\circ} \mathrm{C}\right): 8.0\left(\mathrm{~d}, J_{\mathrm{HH}}=7.8 \mathrm{~Hz}\right.$, $1 \mathrm{H}$, ortho $\mathrm{H}$ of $\mathrm{Ph}), 7.31\left(\mathrm{~d}, J_{\mathrm{HH}}=7.8 \mathrm{~Hz}, 1 \mathrm{H}\right.$, ortho $\mathrm{H}$ of $\left.\mathrm{Ph}\right), 6.90$ $\left(\mathrm{t}, J_{\mathrm{HH}}=7.4,1 \mathrm{H}\right.$, para $\mathrm{H}$ of $\left.\mathrm{Ph}\right), 6.73(\mathrm{~m}, 1 \mathrm{H}$, meta $\mathrm{H}$ of $\mathrm{Ph}), 6.72(\mathrm{~m}$, $1 \mathrm{H}$, meta $\mathrm{H}$ of $\mathrm{Ph}$ ), 1.50 (br, s, $\left.6 \mathrm{H}, \mathrm{PCH}_{3}\right), 1.04$ (vt, $N=13.2 \mathrm{~Hz}$, $\left.18 \mathrm{H}, \mathrm{P}{ }^{t} \mathrm{Bu}\right), 0.76$ (vt, $\left.N=13.2 \mathrm{~Hz}, 18 \mathrm{H}, \mathrm{P}^{\prime} \mathrm{Bu}\right) .{ }^{31} \mathrm{P}\left\{{ }^{1} \mathrm{H}\right\} \mathrm{NMR}(121$ $\left.\mathrm{MHz}, \mathrm{C}_{6} \mathrm{D}_{6}, 20{ }^{\circ} \mathrm{C}\right): 40.5$ (s, Ru-P). ${ }^{19} \mathrm{~F}$ NMR $\left(282 \mathrm{MHz}, \mathrm{C}_{6} \mathrm{D}_{6}, 20\right.$ $\left.{ }^{\circ} \mathrm{C}\right):-77.7\left(\mathrm{~s}, \mathrm{O}_{3} \mathrm{SCF}_{3}\right)$. IR $\left(\mathrm{C}_{6} \mathrm{D}_{6}, \mathrm{~cm}^{-1}\right): v(\mathrm{CO})=1921$.

$\mathbf{R u}(\mathbf{P h})\left(\mathbf{C H}_{3}\right)(\mathbf{C O})\left(\mathbf{P}^{\prime} \mathbf{B u}_{2} \mathbf{M e}\right)_{2},{ }_{15}$. RuPh$(\mathrm{OTf})(\mathrm{CO})\left(\mathrm{P}^{\prime} \mathrm{Bu}_{2} \mathrm{Me}\right)_{2}(200$ $\mathrm{mg}, 0.36 \mathrm{mmol})$ was dissolved in toluene $(10 \mathrm{~mL})$. To the solution, MeLi (1.6 mol/L in diethyl ether, $200 \mu \mathrm{L}, 0.32 \mathrm{mmol})$ was added. The mixture was stirred for $5 \mathrm{~min}$, and the volatiles were evaporated to dryness. The residue was dissolved in tetramethylsilane and filtered. Removal of the solvent results in a viscous oil, which was recrystallized from bis(trimethylsilyl) ether to give orange crystals. Yield: $40 \% .{ }^{1} \mathrm{H}$ NMR $\left(300 \mathrm{MHz}, \mathrm{C}_{6} \mathrm{D}_{6}, 20^{\circ} \mathrm{C}\right): 7.65\left(\mathrm{~d}, J_{\mathrm{HH}}=5.7 \mathrm{~Hz},{ }^{1} \mathrm{H}\right.$ ortho $\mathrm{H}$ of $\mathrm{Ph}), 7.45\left(\mathrm{~d}, J_{\mathrm{HH}}=5.7 \mathrm{~Hz}, 1 \mathrm{H}\right.$, ortho $\mathrm{H}$ of $\left.\mathrm{Ph}\right), 6.72(\mathrm{~m}, 3 \mathrm{H}$, meta and para $\mathrm{H}$ of $\mathrm{Ph}), 1.42\left(\mathrm{vt}, N=12 \mathrm{~Hz}, 18 \mathrm{H}, \mathrm{PCCH}_{3}\right), 1.05\left(\mathrm{t}, J_{\mathrm{PH}}=4 \mathrm{~Hz}\right.$, $\left.3 \mathrm{H}, \mathrm{PCH}_{3}\right), 0.99$ (vt, $N=12 \mathrm{~Hz}, 18 \mathrm{H}, \mathrm{P}\left(\mathrm{C}\left(\mathrm{CH}_{3}\right)_{3}\right) .{ }^{13} \mathrm{C}\left\{{ }^{1} \mathrm{H}\right\} \mathrm{NMR}$ $\left(\mathrm{C}_{7} \mathrm{D}_{8}, 100 \mathrm{MHz}, 2{ }^{\circ} \mathrm{C}\right): 203.7\left(\mathrm{t}, J_{\mathrm{PC}}=13 \mathrm{~Hz}, \mathrm{Ru}-\mathrm{CO}\right), 158.5(\mathrm{~s}$, $\mathrm{Ru}-\mathrm{C}_{\text {ipso }}$ ), 144.0, 139.4, 126.6, 120.4 (s, Ph), 37.5 (vt, $N=15 \mathrm{~Hz}$, $\left.\mathrm{PC}\left(\mathrm{CH}_{3}\right)_{3}\right), 36.4$ (vt, $\left.N=16 \mathrm{~Hz}, \mathrm{PC}\left(\mathrm{CH}_{3}\right)_{3}\right), 30.0,29.5\left(\mathrm{~s}, \mathrm{PC}\left(\mathrm{CH}_{3}\right)_{3}\right)$, $9.5\left(\mathrm{br}, \mathrm{Ru}-\mathrm{CH}_{3}\right), 5.1\left(\mathrm{br}, \mathrm{PCH}_{3}\right)$. IR $\left(\mathrm{C}_{6} \mathrm{D}_{6}, \mathrm{~cm}^{-1}\right)$ : $1883(v(\mathrm{CO}))$. Anal. Calcd for $\mathrm{C}_{26} \mathrm{H}_{50} \mathrm{OP}_{2} \mathrm{Ru}$ : C, 57.64, H, 9.30. Found: C, 57.38, H, 9.48

$\left[\mathbf{R u}(\mathbf{P h})(\mathbf{C O})\left(\mathbf{P}^{t} \mathbf{B u}_{2} \mathrm{Me}_{2}\right] \mathbf{B A r}^{\prime}{ }_{4}, \quad\right.$ 16. $\mathrm{RuPh}(\mathrm{OTf})(\mathrm{CO})\left(\mathrm{P}^{t} \mathrm{Bu}_{2} \mathrm{Me}\right)_{2}$ (150 mg, $0.22 \mathrm{mmol})$ and $\mathrm{NaBAr}_{4}^{\prime}(201 \mathrm{mg}, 0.23 \mathrm{mmol})$ were mixed in fluorobenzene $(5 \mathrm{~mL})$ in a test tube under argon. The mixture was shaken for $10 \mathrm{~min}$ and centrifuged. The liquid was transferred to a Schlenk flask and layered with pentane. After 2 days, red crystals were obtained. Yield: $160 \mathrm{mg}(52 \%)$. Anal. Calcd for $\mathrm{C}_{57} \mathrm{H}_{59} \mathrm{BF}_{24} \mathrm{OP}_{2} \mathrm{Ru}$ : C, 48.59; H, 4.22. Found: C, 48.61; $\mathrm{H}, 4.10 .{ }^{1} \mathrm{H} \mathrm{NMR}\left(\mathrm{CD}_{2} \mathrm{Cl}_{2}, 20\right.$ $\left.{ }^{\circ} \mathrm{C}\right): 7.73$ (s, 8H, BAr $\left.{ }_{4}\right), 7.57$ (s, 4H, BAr $\left.{ }_{4}{ }^{2}\right), 7.15$ (br, s, 2H, Ph), 7.01 (br, s, 2H, Ph), $6.88(\mathrm{~m}, 1 \mathrm{H}$, para $\mathrm{H}$ of $\mathrm{Ph}), 1.22$ (vt, $N=4.8 \mathrm{~Hz}, 6 \mathrm{H}$, $\mathrm{PCH}_{3}$ ), 1.18 (vt, $N=13.2 \mathrm{~Hz}, 18 \mathrm{H}, \mathrm{P}^{t} \mathrm{Bu}$ ), 1.12 (vt, $N=14.4 \mathrm{~Hz}$, $\left.18 \mathrm{H}, \mathrm{P}{ }^{\prime} \mathrm{Bu}\right) .{ }^{19} \mathrm{~F}$ NMR $\left(\mathrm{CD}_{2} \mathrm{Cl}_{2}, 20{ }^{\circ} \mathrm{C}\right):-65.2\left(\mathrm{~s}, \mathrm{BAr}^{\prime}{ }_{4}\right) .{ }^{31} \mathrm{P}\left\{{ }^{1} \mathrm{H}\right\}$ NMR $\left(\mathrm{CD}_{2} \mathrm{Cl}_{2}, 20^{\circ} \mathrm{C}\right)$ : 41.4 (s). IR $\left(\mathrm{CD}_{2} \mathrm{Cl}_{2}\right.$ or fluorobenzene, $\left.\mathrm{cm}^{-1}\right)$ : $v(\mathrm{CO})=1958, v\left(\mathrm{C}-\mathrm{H}_{\text {agostic }}\right)=2722,2672$.

Crystal Structure of $\left[\mathrm{Ru}(\mathrm{Ph})(\mathrm{CO})\left(\mathrm{P}^{t} \mathrm{Bu}_{2} \mathrm{Me}\right)_{2}\right] \mathrm{BAr}^{\prime}{ }_{4}$. X-ray quality crystals were grown from a fluorobenzene/pentane mixture at room
Table 5. Selected Geometric Parameters of $\left[\mathrm{RuPh}(\mathrm{CO})\left(\mathrm{P}^{t} \mathrm{Bu}_{2} \mathrm{Me}\right)_{2}\right]^{+}$

\begin{tabular}{|c|c|c|c|}
\hline \multicolumn{4}{|c|}{ Bond Lengths $(\AA)$} \\
\hline $\mathrm{Ru} 1-\mathrm{P} 10$ & $2.3920(22)$ & Ru1-P20 & $2.3653(28)$ \\
\hline $\mathrm{Ru} 1-\mathrm{C} 4$ & $2.058(12)$ & $\mathrm{Ru} 1-\mathrm{C} 2$ & $1.799(14)$ \\
\hline $\mathrm{C} 2-\mathrm{O} 3$ & $1.163(16)$ & & \\
\hline \multicolumn{4}{|c|}{ Bond Angles (deg) } \\
\hline $\mathrm{P} 10-\mathrm{Ru} 1-\mathrm{P} 20$ & $167.98(14)$ & $\mathrm{P} 10-\mathrm{Ru} 1-\mathrm{C} 2$ & 93.0 \\
\hline $\mathrm{P} 10-\mathrm{Ru} 1-\mathrm{C} 4$ & $96.532(28)$ & $\mathrm{P} 20-\mathrm{Ru} 1-\mathrm{C} 2$ & $95.5(3)$ \\
\hline $\mathrm{P} 20-\mathrm{Ru} 1-\mathrm{C} 4$ & $91.5(3)$ & $\mathrm{C} 2-\mathrm{Ru} 1-\mathrm{C} 4$ & $93.7(8)$ \\
\hline $\mathrm{Ru} 1-\mathrm{P} 10-\mathrm{C} 11$ & $98.1(3)$ & $\mathrm{Ru} 1-\mathrm{P} 10-\mathrm{DFC} 15$ & $126.1(4)$ \\
\hline $\mathrm{Ru} 1-\mathrm{P} 10-\mathrm{C} 19$ & $109.4(4)$ & $\mathrm{Ru} 1-\mathrm{P} 20-\mathrm{C} 29$ & $114.6(5)$ \\
\hline $\mathrm{Ru} 1-\mathrm{P} 20-\mathrm{C} 21$ & $121.7(6)$ & $\mathrm{Ru} 1-\mathrm{P} 20-\mathrm{C} 25$ & $96.6(3)$ \\
\hline
\end{tabular}

temperature. If the crystals were grown at -20 or $-40{ }^{\circ} \mathrm{C}$ in the same solvent system, only twinned crystals were obtained. The highly airsensitive compound was handled in a nitrogen atmosphere glovebag. The crystals were mounted using silicone grease and were then transferred to a goniostat equipped with a nitrogen vapor cold stream at $-170{ }^{\circ} \mathrm{C}$. No decomposition was evident for the crystal at the low temperature. A preliminary automated search for peaks and then analysis using programs DIRAX and TRACER revealed a primitive orthorhombic cell. Following intensity data collection, the only conditions observed were $h=2 n$ for $h 00, k=2 n$ for $0 k 0$, and $l=2 n$ for $00 l$ which uniquely determined space group $P 22_{1} 2_{1} 2_{1}$. Data processing produced a set of 4419 unique intensities and an $R_{\mathrm{av}}=0.098$ for the averaging of 4109 of these which had been observed more than once. Four standards measured every 300 data points had considerable random scatter, but they showed no systematic trends. No correction was made for absorption $\left(\mu=4.2 \mathrm{~cm}^{-1}\right)$. The structure was solved using a combination of direct methods (MULTAN78) and Fourier techniques. The positions of the $\mathrm{Ru}$ atom and the $\mathrm{P}$ and $\mathrm{C}$ atoms bonded to it were obtained from an initial E-map. The positions of the remaining nonhydrogen atoms were obtained from iterations of a least-squares refinement and difference Fourier calculation. Hydrogens were included in fixed calculated positions with thermal parameters fixed at one plus the isotropic thermal parameter of the parent carbon atom. Four of the carbon atoms, $\mathrm{C}(24)$ and $\mathrm{C}(27)$ in a tert-butyl group and $\mathrm{C}(75)$ and $\mathrm{C}(77)$ in the anion, had thermal parameters that refined to nonpositive definite anisotropic values. In the final cycles of refinement, these four atoms were varied with isotropic thermal parameters and the remaining 82 non-hydrogen atoms were varied with anisotropic thermal parameters to give a final $R(F)=0.062$ for the 756 total variables (Tables 1 and 5). The largest peak in the final difference map was 0.95 , and the deepest hole was -1.15 e/ $\AA^{3}$.

Reaction of $\left[\mathrm{RuPh}(\mathrm{CO})\left(\mathrm{P}^{t} \mathrm{Bu}_{2} \mathrm{Me}\right)_{2}\right] \mathrm{BAr^{ \prime }}{ }_{4}$ with $\mathrm{C}_{6} \mathrm{H}_{4} \mathrm{O}_{2} \mathrm{~B}-\mathrm{H}$ $\left[\mathrm{RuPh}(\mathrm{CO})\left(\mathrm{P}^{t} \mathrm{Bu}_{2} \mathrm{Me}\right)_{2}\right] \mathrm{BAr}_{4}^{\prime}\left(10 \mathrm{mg}, 7.2 \times 10^{-3} \mathrm{mmol}\right)$ and catecholborane $\left(0.76 \mu \mathrm{L}, 7.2 \times 10^{-3} \mathrm{mmol}\right)$ were mixed in $\mathrm{CD}_{2} \mathrm{Cl}_{2}(0.5$ $\mathrm{mL}$ ). After $1 \mathrm{~h}$, NMR analysis of the reaction solution reveals the formation of $\left[\mathrm{RuH}(\mathrm{CO})\left(\mathrm{P}^{t} \mathrm{Bu}_{2} \mathrm{Me}\right)_{2}\right]^{+}$and $\mathrm{C}_{6} \mathrm{H}_{4} \mathrm{O}_{2} \mathrm{~B}-\mathrm{Ph}$, which was confirmed by comparing the NMR spectra with authentic samples.

$\mathbf{R u}\left(\mathbf{B O}_{2} \mathbf{C}_{6} \mathbf{H}_{4}\right)(\mathbf{O T f})(\mathbf{C O})\left(\mathbf{P}^{t} \mathbf{B u}_{2} \mathbf{M e}\right)_{2}$, 17. $\mathrm{RuH}(\mathrm{OTf})(\mathrm{CO})\left(\mathrm{P}^{t} \mathrm{Bu}_{2} \mathrm{Me}\right)_{2}$ $\left(0.50 \mathrm{~g}, 8.3 \times 10^{-4} \mathrm{~mol}\right)$ and catecholborane $\left(97 \mu \mathrm{L}, 1.0 \times 10^{-3} \mathrm{mmol}\right)$ were mixed with $\mathrm{C}_{6} \mathrm{H}_{6}(10 \mathrm{~mL})$ and heated at $80^{\circ} \mathrm{C}$ for $4 \mathrm{~h}$. The mixture was evaporated to give a yellow solid, which was recrystallized from pentane/benzene mixture to give light-yellow crystals. Yield: $0.40 \mathrm{~g}$ $(67 \%)$. Anal. Calcd for $\mathrm{C}_{26} \mathrm{H}_{40} \mathrm{BF}_{3} \mathrm{O}_{6} \mathrm{P}_{2} \mathrm{RuS} ; \mathrm{C}, 43.50, \mathrm{H}, 5.62$. Found: $\mathrm{C}, 44.01, \mathrm{H}, 6.64 .{ }^{1} \mathrm{H}$ NMR $\left(300 \mathrm{MHz}, \mathrm{C}_{6} \mathrm{D}_{6}, 20{ }^{\circ} \mathrm{C}\right): 7.00(\mathrm{~m}, 2 \mathrm{H}$, $\left.\mathrm{O}_{2} \mathrm{C}_{6} \mathrm{H}_{4}\right), 6.70\left(\mathrm{~m}, 2 \mathrm{H}, \mathrm{O}_{2} \mathrm{C}_{6} \mathrm{H}_{4}\right) 1.57\left(\mathrm{vt}, N=5.9 \mathrm{~Hz}, 6 \mathrm{H}, \mathrm{PCH}_{3}\right), 1.12$ (vt, $\left.N=13.3 \mathrm{~Hz}, 18 \mathrm{H}, \mathrm{PC}\left(\mathrm{CH}_{3}\right)_{3}\right), 0,85$ (vt, $N=13 \mathrm{~Hz}, 18 \mathrm{H}, \mathrm{PC}-$ $\left.\left(\mathrm{CH}_{3}\right)_{3}\right) .{ }^{31} \mathrm{P}\left\{{ }^{1} \mathrm{H}\right\}$ NMR $\left(\mathrm{C}_{6} \mathrm{D}_{6}, 20{ }^{\circ} \mathrm{C}\right): 53.6\left(\mathrm{~s}, w_{1 / 2}=194 \mathrm{~Hz}\right){ }^{19} \mathrm{~F}$ NMR $\left(\mathrm{C}_{6} \mathrm{D}_{6}, 20{ }^{\circ} \mathrm{C}\right):-78.6\left(\mathrm{~s}, \mathrm{CF}_{3}\right) .{ }^{11} \mathrm{~B}$ NMR $\left(\mathrm{C}_{6} \mathrm{D}_{6}, 20{ }^{\circ} \mathrm{C}\right): 44.5$ (br s). IR $\left(\mathrm{C}_{6} \mathrm{D}_{6}, \mathrm{~cm}^{-1}\right)$ : $1939(v(\mathrm{CO}))$.

$\left[\mathbf{R u}\left(\mathrm{BO}_{2} \mathrm{C}_{6} \mathrm{H}_{4}\right)(\mathbf{C O})\left(\mathrm{P}^{t} \mathrm{Bu}_{2} \mathrm{Me}\right)_{2}\right]$, 18. $\mathrm{Ru}\left(\mathrm{BO}_{2} \mathrm{C}_{6} \mathrm{H}_{4}\right)(\mathrm{OTf})(\mathrm{CO})-$ $\left(\mathrm{P}^{t} \mathrm{Bu}_{2} \mathrm{Me}\right)_{2}(10 \mathrm{mg} .0 .014 \mathrm{mmol})$ and $\mathrm{NaBAr}_{4}{ }_{4}(12.4 \mathrm{mg}, 0.014 \mathrm{mmol})$ were mixed in $\mathrm{CD}_{2} \mathrm{Cl}_{2}$ to give a yellow solution. NMR analysis of the mixture revealed clean formation of $\left[\mathrm{Ru}\left(\mathrm{BO}_{2} \mathrm{C}_{6} \mathrm{H}_{4}\right)(\mathrm{CO})\left(\mathrm{P}^{t} \mathrm{Bu}_{2} \mathrm{Me}\right)_{2}\right]-$ BAr ${ }_{4}{ }^{1}{ }^{1} \mathrm{H}$ NMR: 7.73 (s, 8H, ortho $\mathrm{H}$ of $\left.\mathrm{Ar}^{\prime}\right), 7.57$ (s, $4 \mathrm{H}$, para $\mathrm{H}$ of $\left.\mathrm{Ar}^{\prime}\right), 7.21\left(\mathrm{~m}, 2 \mathrm{H}, \mathrm{O}_{2} \mathrm{C}_{6} \mathrm{H}_{4}\right), 7.05\left(2 \mathrm{H}, \mathrm{O}_{2} \mathrm{C}_{6} \mathrm{H}_{4}\right), 1.44$ (vt, $N=5.1 \mathrm{~Hz}$, $\left.6 \mathrm{H}, \mathrm{PCH}_{3}\right), 1.21$ (vt, $\left.N=13.5 \mathrm{~Hz}, 38 \mathrm{H}, \mathrm{PC}\left(\mathrm{CH}_{3}\right)_{3}\right), 1.21$ (vt, $N=$ 
13.5 Hz, $\left.18 \mathrm{H}, \mathrm{PC}\left(\mathrm{CH}_{3}\right)_{3}\right) .{ }^{31} \mathrm{P}\left\{{ }^{1} \mathrm{H}\right\}$ NMR: 43.3 (br s), ${ }^{19} \mathrm{~F}$ NMR: -64.1 (s). ${ }^{11} \mathrm{~B}$ NMR: 42.8 (br,s). IR: $1981(v(\mathrm{CO}))$.

Reaction of $\mathrm{Ru}(\mathrm{Ph})(\mathrm{OTf})(\mathrm{CO})\left(\mathrm{P}^{t} \mathrm{Bu}_{2} \mathrm{Me}\right)_{2}$ with Catecholborane. $\mathrm{RuPh}(\mathrm{OTf})(\mathrm{CO})\left(\mathrm{P}^{t} \mathrm{Bu}_{2} \mathrm{Me}\right)_{2}(10 \mathrm{mg}, 0.015 \mathrm{mmol})$ and catecholborane $(2.0 \mu \mathrm{L}, 0.023 \mathrm{mmol})$ were mixed in $\mathrm{C}_{6} \mathrm{D}_{6}(0.5 \mathrm{~mL})$ and heated at 80 ${ }^{\circ} \mathrm{C}$ for $8 \mathrm{~h} .{ }^{1} \mathrm{H}$ NMR spectroscopic analysis revealed formation of $\mathrm{RuH}-$ $(\mathrm{OTf})(\mathrm{CO})\left(\mathrm{P}^{\prime} \mathrm{Bu}_{2} \mathrm{Me}\right)_{2}$ and $\mathrm{Ru}\left(\mathrm{BO}_{2} \mathrm{C}_{6} \mathrm{H}_{4}\right)(\mathrm{OTf})(\mathrm{CO})\left(\mathrm{P}^{t} \mathrm{Bu}_{2} \mathrm{Me}\right)_{2}$ with a ca. 1:1 ratio along with $\mathrm{PhBO}_{2} \mathrm{C}_{6} \mathrm{H}_{4}$. If one more equivalent of catecholborane was added and heated for $4 \mathrm{~h}$, only $\mathrm{Ru}\left(\mathrm{BO}_{2} \mathrm{C}_{6} \mathrm{H}_{4}\right)(\mathrm{OTf})-$ $(\mathrm{CO}) \mathrm{L}_{2}$ was observed.

Reaction of $\left[\mathrm{Ru}(\mathrm{Ph})(\mathrm{CO})\left(\mathrm{P}^{t} \mathrm{Bu}_{2} \mathrm{Me}\right)_{2}\right] \mathrm{BAr}_{4}^{\prime}$ with $\mathrm{Me}_{3} \mathrm{SiCCH}$. When $\left[\mathrm{RuPh}(\mathrm{CO})\left({ }^{t} \mathrm{Bu}_{2} \mathrm{Me}_{2}\right)_{2}\right] \mathrm{BAr}_{4}{ }_{4}\left(10 \mathrm{mg}, 7.6 \times 10^{-3} \mathrm{mmol}\right)$ and $\mathrm{Me}_{3-}$ SiCCH $\left(1 \mu \mathrm{L}, 7.6 \times 10^{-3} \mathrm{mmol}\right)$ were dissolved in $\mathrm{CD}_{2} \mathrm{Cl}_{2}$, the solution color changed to red immediately. NMR analysis revealed partial consumption of $\left[\mathrm{RuPh}(\mathrm{CO})\left({ }^{t} \mathrm{Bu}_{2} \mathrm{Me}\right)_{2}\right] \mathrm{BAr}_{4}^{\prime}$ and formation of $\left[\mathrm{Ru}\left(\left(\mathrm{Me}_{3}-\right.\right.\right.$ $\left.\mathrm{SiCH}=\mathrm{C}-\mathrm{CH}=\mathrm{CH}\left(\mathrm{SiMe}_{3}\right)(\mathrm{CO})\left(\mathrm{P}^{t} \mathrm{Bu}_{2} \mathrm{Me}\right)_{2}\right] \mathrm{BAr}_{4}{ }_{4}{ }^{11}$ and trace $[\mathrm{Ru}-$ $\left.\left(\mathrm{CH}=\mathrm{CHSiMe}_{3}\right)(\mathrm{CO})\left(\mathrm{P}^{t} \mathrm{Bu}_{2} \mathrm{Me}\right)_{2}\right] \mathrm{BAr}_{4}{ }_{4}{ }^{1} \mathrm{H}$ NMR $\left(300 \mathrm{MHz}, \mathrm{CD}_{2} \mathrm{Cl}_{2}\right.$, $\left.20{ }^{\circ} \mathrm{C}\right): 7.71\left(\mathrm{~s}, 8 \mathrm{H}\right.$, ortho $\mathrm{H}$ of $\left.\mathrm{Ar}^{\prime}\right), 7.51\left(\mathrm{~d}, J_{\mathrm{HH}}=12.3 \mathrm{~Hz}, 1 \mathrm{H}\right.$, $\mathrm{RuCH}), 5.50\left(\mathrm{dt}, J_{\mathrm{HH}}=12.3 \mathrm{~Hz}, J_{\mathrm{PH}}=2 \mathrm{~Hz}, 1 \mathrm{H}, \mathrm{RuCH}=\mathrm{CH}\right), 1.39$ (vt, $\left.N=4.5 \mathrm{~Hz}, 6 \mathrm{H}, \mathrm{PCH}_{3}\right), 1.28\left(\mathrm{vt}, N=13.2 \mathrm{~Hz}, 18 \mathrm{H}, \mathrm{P}{ }^{t} \mathrm{Bu}\right), 1.19$ (vt, $\left.N=13.2 \mathrm{~Hz}, 18 \mathrm{H}, \mathrm{P} / \mathrm{Bu}) .0 .038\left(\mathrm{~s}, 9 \mathrm{H}, \mathrm{SiCH}_{3}\right)_{3}\right) .{ }^{31} \mathrm{P}\left\{{ }^{1} \mathrm{H}\right) \mathrm{NMR}$ (121 MHz, $\mathrm{CD}_{2} \mathrm{Cl}_{2}, 20^{\circ} \mathrm{C}$ ): $\delta 40.8$ (s). IR (Nujol, $\mathrm{cm}^{-1}$ ): 2726, 2677 $\left(v\left(\mathrm{C}-\mathrm{H}_{\text {agostic }}\right)\right), 1944(v(\mathrm{CO}))$. If two more equivalents of $\mathrm{Me}_{3} \mathrm{SiCCH}$ are added, clean formation of $\left[\mathrm{Ru}\left(\left(\mathrm{Me}_{3} \mathrm{SiCH}=\mathrm{C}-\mathrm{CH}=\mathrm{CH}\left(\mathrm{SiMe}_{3}\right)-\right.\right.\right.$ $\left.(\mathrm{CO})\left(\mathrm{P}^{\prime} \mathrm{Bu}_{2} \mathrm{Me}\right)_{2}\right] \mathrm{BAr}_{4}{ }_{4}$ was achieved. Analysis of the volatiles revealed the presence of $\mathrm{PhCCSiMe}_{3}$ as the only product that contains the $\mathrm{Ph}$ group

General Procedure for Low-Temperature NMR Spectroscopic Study. $\left[\mathrm{Ru}(\mathrm{Ph})(\mathrm{CO})\left(\mathrm{P}^{t} \mathrm{Bu}_{2} \mathrm{Me}\right)_{2}\right] \mathrm{BAr}_{4}{ }_{4}(10 \mathrm{mg})$ was placed in an NMR tube with Teflon valve closure and carefully covered with $\mathrm{CD}_{2} \mathrm{Cl}_{2}(0.5$ $\mathrm{mL}$ ) so that the crystals were settled at the bottom of the tube. To the headspace of the tube, $\mathrm{Me}_{3} \mathrm{SiCCH}(1 \mu \mathrm{L})$ was added. The tube was then promptly taken out of glovebox and placed in a dry ice acetone bath. The tube was then shaken thoroughly and transferred to a precooled NMR probe for observation.

\section{Results and Discussion}

Structure of $\mathbf{R u H C l}(\mathbf{C O})\left(\mathbf{P i P r}_{3}\right)_{2}$, 1. This complex was originally synthesized by Werner and co-workers. ${ }^{12}$ The chemical reactivity has been extensively studied mainly by Esteruelas and co-workers. ${ }^{13}$ The rich reactivity ranges from highly regioselective hydrosilation catalysis to synthesis of cyclopentadienyl complexes such as $\mathrm{CpRuCl}(\mathrm{CO})\left(\mathrm{P}^{\mathrm{i}} \mathrm{Pr}_{3}\right)$. Compounds with different phosphine ligands $\left(\mathrm{PCy}_{3}\right.$ and $\left.\mathrm{P}^{t} \mathrm{Bu}_{2} \mathrm{Me}\right)$ have also been synthesized by a similar method. ${ }^{14}$ The structure assignment of 1 was based solely on spectroscopic data. These data, however, do not provide any information on the weak interactions such as agostic bonding. Therefore, we carried out the $\mathrm{X}$-ray single-crystal structure determination of $\mathbf{1}$. The ORTEP plot (Figure 1) shows a square-based pyramidal geometry with hydride trans to the vacant site and the $\pi$-donor $(\mathrm{Cl})$ and the $\pi$-acceptor ( $\mathrm{CO}$ ) ligands trans to each other, benefiting from push-pull stabilization. Although there is disorder around a center of symmetry, all three $\mathrm{Ru}-\mathrm{P}-\mathrm{C}$ (methine) angles are normal (around $\left.113^{\circ}\right)$ and the shortest distance from $\mathrm{Ru}$ to $\left(\mathrm{CH}_{3}\right)$ carbon $(3.5 \AA)$ is too long for agostic interaction. Therefore, $\mathbf{1}$ is authentically coordinatively unsaturated. Other features of the structure are normal for $\mathrm{Ru}(\mathrm{II})$ complexes and deserve no further

(11) Huang, D.; Oliván, M.; Huffman, J. C.; Eisenstein, O.; Caulton, K. G. Organometallics 1998, 17, 4700.

(12) Esteruelas, M. A.; Werner, H. J. Organomet. Chem. 1986, 303, 221.

(13) (a) Esteruelas, M. A.; Juana, H.; Oro, L. A. Organometallics 1993 , 14, 2377, (b) Esteruelas, M. A.; Gomez, A. V.; Lahoz, F. J.; Ana, M.; Onate, E.; Oro, L. A. Organometallics 1996, 15, 3423.

(14) Moers, F. G.; Langhout, J. P. Recl. Trav. Chim. Pays-Bas 1972 $91,591$.

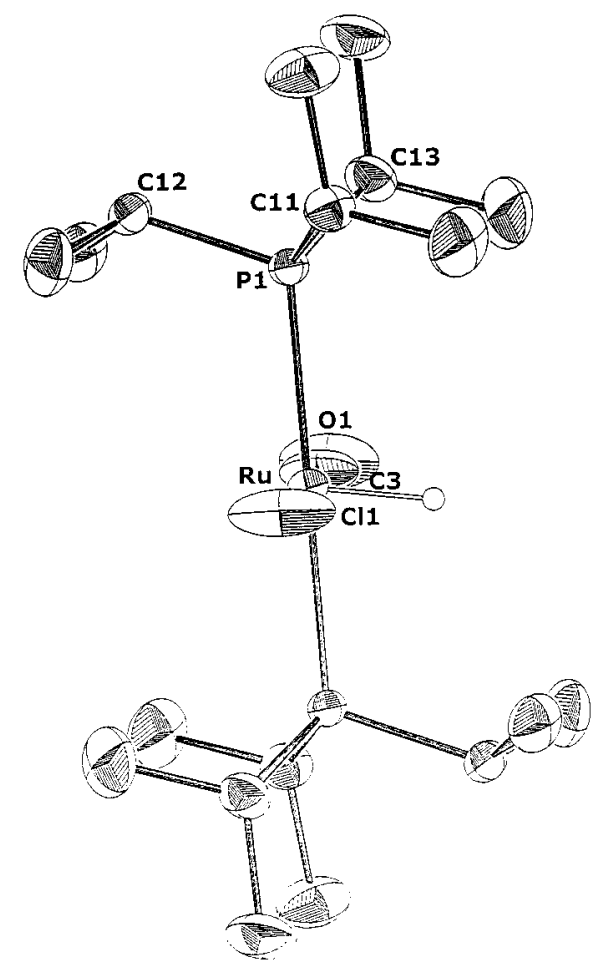

Figure 1. ORTEP diagram (50\% probability level) of $\mathrm{RuHCl}(\mathrm{CO})$ $\left(\mathrm{PiPr}_{3}\right)_{2}, 1$. Hydrogen atoms are omitted except those bound to Ru.

comment. The structure of $\mathbf{1}$ is similar with that of Os analogue $\mathrm{OsHCl}(\mathrm{CO})\left(\mathrm{PCy}_{3}\right)_{2}$, which also has no agostic interaction. ${ }^{15}$

Synthesis and X-ray Crystal Structure of $\mathrm{RuCl}_{2}(\mathrm{CO}) \mathrm{L}_{2}$ ( $\left.\mathbf{L}=\mathbf{P i P r}_{\mathbf{3}}\right)$, 2. The hydride in $\mathbf{1}$ is replaced by chloride using $\mathrm{PhCH}_{2} \mathrm{Cl}\left(80{ }^{\circ} \mathrm{C}, 4 \mathrm{~h}\right)$ to give 2 in good yield (eq 1). The

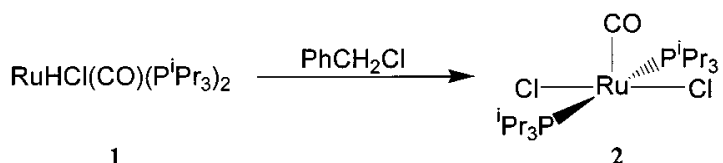

complex has been reported as a byproduct (12\% yield) in the synthesis of $1 .^{16}$ The method reported here gives a rational synthesis, which is also a quite specific one, since benzyl chloride does not transform $\operatorname{RuHCl}(\mathrm{CO})\left(\mathrm{P}^{t} \mathrm{Bu}_{2} \mathrm{Me}\right)_{2}$ to $\mathrm{RuCl}_{2}(\mathrm{CO})\left(\mathrm{P}^{t} \mathrm{Bu}_{2} \mathrm{Me}\right)_{2}$, 3. The latter has been synthesized by reaction with $\mathrm{CHCl}_{3}$, and the reactivity has been reported. ${ }^{7 \mathrm{~b}}$ The spectroscopic data of $\mathbf{2}$ includes only one virtual triplet of doublets for the methyl group, indicative of the two symmetry elements to make all $\mathrm{CH}_{3}$ of ${ }^{i} \mathrm{Pr}$ magnetically equivalent. Moreover, the much higher CO stretching frequency (1937 vs $1908 \mathrm{~cm}^{-1}$ of 1) suggests geometry differences between them. These results support the geometry with $\mathrm{CO}$ trans to the vacant site, which is proved by X-ray single-crystal structure analysis. The ORTEP plot of $\mathbf{2}$ is depicted in Figure 2 and the geometric parameters are collected in Table 3. Like 1, 2 also adopts a square-based pyramidal geometry, but with two mutually trans chlorides and phosphines at the basal and $\mathrm{CO}$ at the apical site. This arrangement is in agreement with the computational prediction on the geometry preference of five-coordinate $d^{6}$ metal complexes; the highest trans influence ligand occupies the apical site, which raises the energy of the LUMO. In 2, the

(15) Moers, F. G.; Nordik, J. H.; Beurskens, P. T. Cryst. Struct. Comm. 1981, 10, 1149 .

(16) Werner H.; Tena, M. A.; Mahr, N.; Peters, K.; von Schnering, H. G. Chem. Ber. 1995, 128, 41 . 


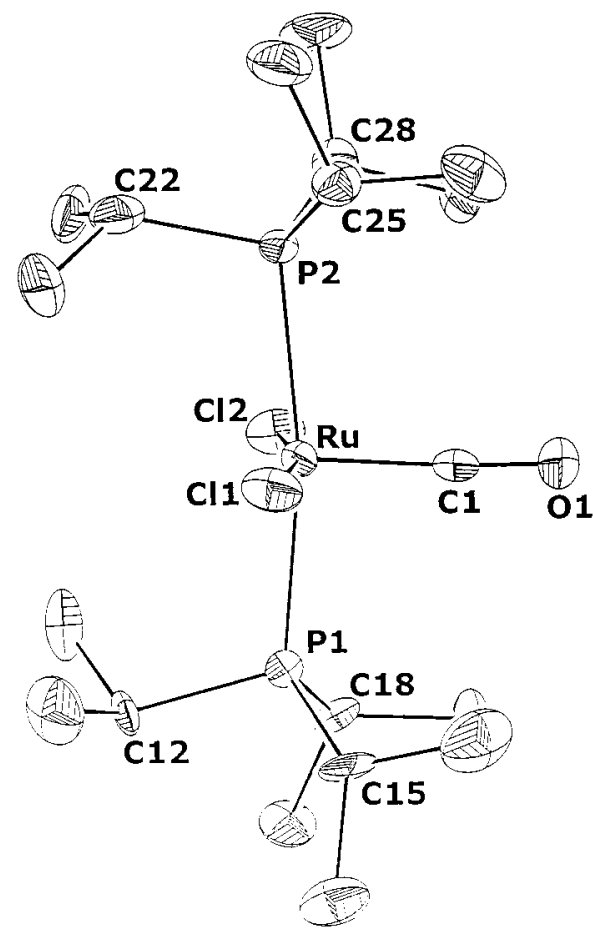

Figure 2. ORTEP diagram (50\% probability level) of $\mathrm{RuCl}_{2}(\mathrm{CO})$ $\left(\mathrm{PiPr}_{3}\right)_{2}, 2$. Hydrogen atoms are omitted.

$\mathrm{P}-\mathrm{Ru}-\mathrm{P}$ and $\mathrm{Cl}-\mathrm{Ru}-\mathrm{Cl}$ angles significantly deviate from $180^{\circ}$. A similar structure is adopted by $\mathrm{RuCl}_{2}(\mathrm{CHR}) \mathrm{L}_{2} \cdot{ }^{17}$ The bending of $\mathrm{Cl}-\mathrm{Ru}-\mathrm{Cl}$ suppresses filled-filled repulsion between the $\mathrm{Cl}$ lone pair and the metal $\mathrm{d} \pi$ electron. One ${ }^{i} \mathrm{Pr}$ group of phosphine bends toward to the vacant site so that the $\mathrm{Ru}-$ $\mathrm{P} 2-\mathrm{C} 22$ is as much as $10^{\circ}$ smaller than that of $\mathrm{Ru}-\mathrm{P} 2-\mathrm{C} 28$ (or C25). This may indicate weak agostic interaction, since all corresponding angles in nonagostic $\mathbf{1}$ are nearly identical. However, the long distance of $\mathrm{Ru}$ to the nearest $\mathrm{CH}_{3}$ carbon $(3.6 \AA)$ and $\mathrm{Ru} / \mathrm{H}(2.98 \AA)$ speaks against any bonding interactions. This is in marked contrast with $\mathrm{RuCl}_{2}(\mathrm{CO})\left(\mathrm{PCy}_{3}\right)_{2}$, which has an agostic interaction between ortho $\mathrm{CH}_{2}$ of cyclohexyl and $\mathrm{Ru}(\mathrm{II})(\mathrm{Ru} / \mathrm{C}$ is $3.0 \AA$, and $\mathrm{Ru} / \mathrm{H}$ is $2.3 \AA) .{ }^{18}$ For comparison, in $\mathrm{Rh}($ mesityl) 3 , distances to agostic ortho methyl groups are $\mathrm{Rh} / \mathrm{C}=2.8 \AA$ and $\mathrm{Rh} / \mathrm{H}=2.25-2.37 \AA .{ }^{19}$ The interplay of steric effects and the trans influence has been addressed in recent theoretical calculations. ${ }^{20}$

$\mathrm{Ru}(\mathrm{Me}) \mathrm{Cl}(\mathrm{CO}) \mathrm{L}_{2}$ and $\mathrm{RuMe}_{2}(\mathrm{CO}) \mathrm{L}_{2}$. Addition of 1 equiv of MeLi to $\mathbf{2}$ in toluene results in immediate formation of equal amounts of $\mathrm{RuMeCl}(\mathrm{CO})\left(\mathrm{P}^{i} \mathrm{Pr}_{3}\right)_{2}, \mathbf{4}$, and $\mathrm{RuMe}_{2}(\mathrm{CO})\left(\mathrm{P}^{i} \mathrm{Pr}_{3}\right)_{2}$, 6, along with some starting materials (Scheme 1). After $3 \mathrm{~h}, 4$ is the dominant product $(>90 \%)$ with a small amount of starting material. Apparently, ligand redistribution between $\mathbf{2}$ and $\mathbf{6}$ occurs. Ligand scrambling of similar complexes has been examined before and is considered to be an associative process even though there are two sterically demanding phosphine ligands. ${ }^{21}$ If one more equivalent of $\mathrm{MeLi}$ is added, clean conversion to 6 results. The reaction also succeeds with the $\mathrm{P}^{t} \mathrm{Bu}_{2} \mathrm{Me}$ analogue. $\mathrm{RuMeCl}(\mathrm{CO})\left(\mathrm{P}^{t} \mathrm{Bu}_{2} \mathrm{Me}\right)_{2}, \mathbf{5}$, has been syn-

(17) Schwab, P.; Grubbs, R. H.; Ziller, J. W. J. Am. Chem. Soc. 1996, $118,100$.

(18) Moers, F. G.; Beurskens, P. T.; Noordik, J. H. Cryst. Struct. Commun. 1982, 11, 1655 .

(19) Hay-Motherwell, R. S.; Koschmieder, S. U.; Wilkinson, G.; HussainBaites, B.; Hursthouse, M. B. J. Chem. Soc., Dalton Trans. 1991, 2821.

(20) Ujaque, G.; Cooper, A. C.; Maseras, F.; Eisenstein, O.; Caulton, K. G. J. Am. Chem. Soc. 1998, 120, 361 .

(21) Poulton, J. T.; Hauger, B. E.; Kuhlman, R.; Caulton, K. G. Inorg. Chem. 1994, 33, 3325. thesized independently by treatment of $\mathrm{RuHCl}(\mathrm{CO})\left(\mathrm{P}^{t} \mathrm{Bu}_{2} \mathrm{Me}\right)_{2}$ with diazomethane, but this reaction fails to convert 1 to $4^{7 \mathrm{a}}$ The CO stretching frequency $\left(1898 \mathrm{~cm}^{-1}\right)$ of $\mathbf{5}$ is close to that of $\mathrm{RuHCl}(\mathrm{CO})\left(\mathrm{P}^{t} \mathrm{Bu}_{2} \mathrm{Me}\right)_{2}\left(1906 \mathrm{~cm}^{-1}\right)$, suggesting that they have similar geometry. Since methyl has the strongest trans influence among the ligands, one may reasonably assume that the complex has a square-based pyramidal geometry with $\mathrm{Me}$ trans to the vacant site. In agreement with this, the carbon resonance of the $\mathrm{Ru}-\mathrm{CH}_{3}$ of $\mathbf{5}$ appears at unusually high field $(-11.0 \mathrm{ppm})$. Similarly, the (Os) $-\mathrm{CH}_{3}{ }^{13} \mathrm{C}\left\{{ }^{1} \mathrm{H}\right\}$ NMR resonance of $\mathrm{Os}\left(\mathrm{CH}_{3}\right) \mathrm{Cl}(\mathrm{CO})\left(\mathrm{P}^{t} \mathrm{Bu}_{2} \mathrm{Me}\right)_{2}$ appears at unusually high field $(-38 \mathrm{ppm}) .{ }^{7 \mathrm{a}}$ The high field shift of the hydride has been a diagnostic feature of five-coordinate $\mathrm{Ru}$ and Os complexes with hydride trans to the vacant site, and perhaps the same is true of the ${ }^{13} \mathrm{C}$ chemical shift in such a site. The dimethyl complex $\mathrm{RuMe}_{2}(\mathrm{CO})\left(\mathrm{P}^{t} \mathrm{Bu}_{2} \mathrm{Me}\right)_{2}, 7$, shows only one ruthenium methyl proton (or carbon) resonance at room temperature as well as one ${ }^{t} \mathrm{Bu}$ signal, indicating that the two $(\mathrm{Ru})-\mathrm{CH}_{3}$ and all the ${ }^{t} \mathrm{Bu}$ methyls are equivalent. However, at $-90{ }^{\circ} \mathrm{C}$, two ${ }^{1} \mathrm{H}$ and ${ }^{13} \mathrm{C}\left\{{ }^{1} \mathrm{H}\right\} \mathrm{Ru}-\mathrm{CH}_{3}$ signals $\left({ }^{13} \mathrm{C},-15.7\right.$ and $\left.17.5 \mathrm{ppm}\right)$ are observed along with two ${ }^{t} \mathrm{Bu}$ peaks $\left({ }^{1} \mathrm{H}\right.$ or $\left.{ }^{13} \mathrm{C}\right)$. Therefore, the ground-state geometry of the complex has one methyl in the apical site and the other trans to $\mathrm{CO}$ in the basal plane. On the basis of the decoalescence temperature of the ${ }^{13} \mathrm{C}$ signals of the $\mathrm{Ru}-\mathrm{CH}_{3}$, the energy barrier $\left(\Delta G^{\ddagger}\right)$ of the conversion is calculated as $6.8 \mathrm{kcal} / \mathrm{mol}$ at $-30{ }^{\circ} \mathrm{C}$. Since it is an intramolecular process, the entropy change $\left(\Delta S^{\ddagger}\right)$ is expected to be small such that the $\Delta G^{\ddagger}$ value is close to that of $\Delta H^{\ddagger}$. The hydride site exchange of $\mathrm{Ru}(\mathrm{H})_{2}(\mathrm{CO})\left(\mathrm{P}^{t} \mathrm{Bu}_{2} \mathrm{Me}\right)_{2}$ has the $\Delta H^{\ddagger}$ of 7.6 $\mathrm{kcal} / \mathrm{mol}$ and $\Delta S^{\ddagger}$ of $6.5 \mathrm{eu}$, so $\Delta G^{\ddagger}$ for $\mathrm{Ru}(\mathrm{H})_{2}(\mathrm{CO}) \mathrm{L}_{2}$ at -30 ${ }^{\circ} \mathrm{C}$ is $6.0 \mathrm{kcal} / \mathrm{mol}^{22}$ This number compares well to the barrier of $\mathrm{Ru}\left(\mathrm{CH}_{3}\right)_{2}(\mathrm{CO})\left(\mathrm{P}^{t} \mathrm{Bu}_{2} \mathrm{Me}\right)_{2}$, indicating a similar process for methyl site exchange as that of hydride site exchange of Ru$(\mathrm{H})_{2}(\mathrm{CO}) \mathrm{L}_{2}$, which is calculated to go through an intermediate with $\mathrm{CO}$ trans to the vacant site and the two hydride trans to each other (eq 2).

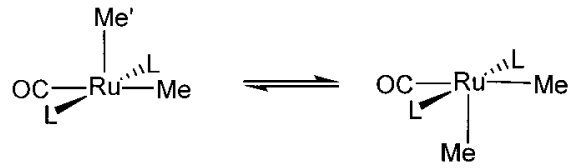

Synthesis of RuMeF(CO) $\mathrm{L}_{2}$, RuMe(OTf)(CO) $\mathrm{L}_{2}$, RuMe$\left(\mathrm{BF}_{4}\right)(\mathbf{C O}) \mathbf{L}_{2}$, and $\left[\operatorname{RuMe}(\mathbf{C O}) \mathbf{L}_{2}\right] \mathbf{B A r}^{\prime}{ }_{4}$. Halide exchange of $\mathrm{RuMeCl}(\mathrm{CO})\left(\mathrm{P}^{t} \mathrm{Bu}_{2} \mathrm{Me}\right)_{2}$ with $\mathrm{CsF}$ in acetone for $4 \mathrm{~h}$ gives $\mathrm{RuMeF}(\mathrm{CO}) \mathrm{L}_{2}, 8$ (Scheme 1), in quantitative yield as judged by NMR and in $54 \%$ isolated yield. The complex is characterized by the doublet of the ${ }^{31} \mathrm{P} \mathrm{NMR}$ signal $\left(J_{\mathrm{PF}}=22 \mathrm{~Hz}\right)$ and a triplet of quartets of the ${ }^{19} \mathrm{~F}$ resonance $\left(-201 \mathrm{ppm}, J_{\mathrm{PF}}=22\right.$ $\mathrm{Hz}, J_{\mathrm{HF}}=4 \mathrm{~Hz}$ ). The fluoride readily reacts with 1 equiv of $\mathrm{BF}_{3} \cdot \mathrm{OEt}_{2}\left(\right.$ in $\mathrm{Et}_{2} \mathrm{O}$ ) to give $\mathrm{RuMe}\left(\mathrm{BF}_{4}\right)(\mathrm{CO}) \mathrm{L}_{2}, \mathbf{1 0}$ (Scheme 1), which is not soluble in diethyl ether and precipitates from the reaction solution. However, it is soluble in benzene or toluene, thus $\mathrm{BF}_{4}{ }^{-}$is likely to be coordinating. Consistent with this, the ${ }^{19} \mathrm{~F}$ NMR spectrum of the $\mathrm{BF}_{4}$ is an extremely broad peak $(-210$ ppm, $\omega_{1 / 2}=1396 \mathrm{~Hz}$ ) at $20^{\circ} \mathrm{C}$, due to exchange of coordinated $\mathrm{BF}_{4}{ }^{-}$. The $\mathrm{CO}$ stretching frequency of $\mathbf{1 0}$ is higher than that of the $\mathrm{Ru}(\mathrm{Me}) \mathrm{Cl}(\mathrm{CO}) \mathrm{L}_{2}\left(1919\right.$ vs $\left.1898 \mathrm{~cm}^{-1}\right)$. The fluoride in $\mathbf{8}$ is also readily replaced by trifluoromethane sulfonate (triflate) using $\mathrm{Me}_{3} \mathrm{SiOTf}$ (diethyl ether, $10 \mathrm{~min}$ ) to give $\mathrm{RuMe}(\mathrm{OTf}$ )$(\mathrm{CO}) \mathrm{L}_{2}, 9$ (Scheme 1). The $\mathrm{CO}$ stretching frequency of this complex is higher $\left(1914 \mathrm{~cm}^{-1}\right)$ than that of $\mathbf{4}$, in accordance

(22) Heyn, R. H.; Macgregor, S. A.; Nadasdi, T. T.; Ogasawara, M.; Eisenstein, O. Caulton, K. G. Inorg. Chim. Acta 1997, 259, 5. 


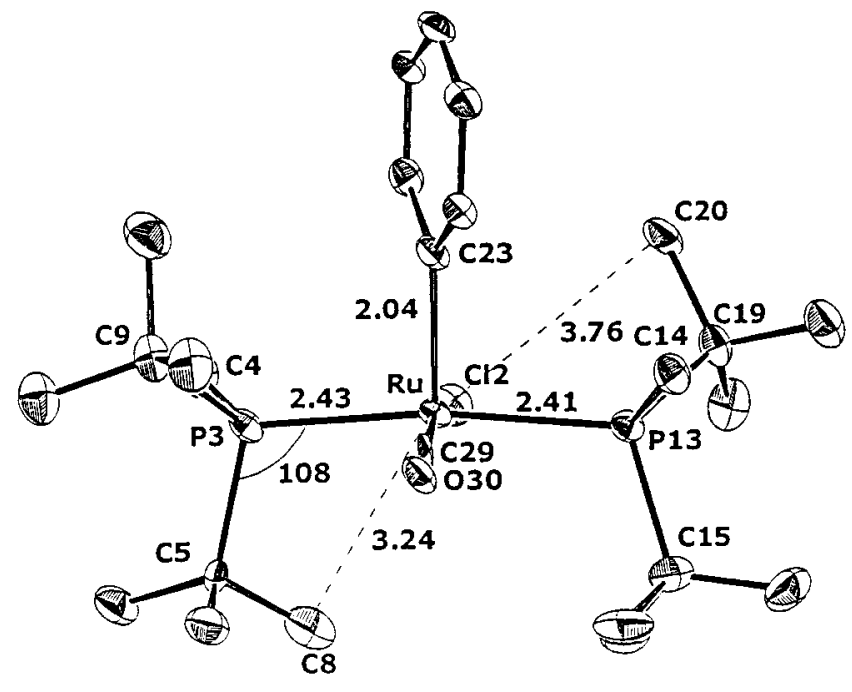

a

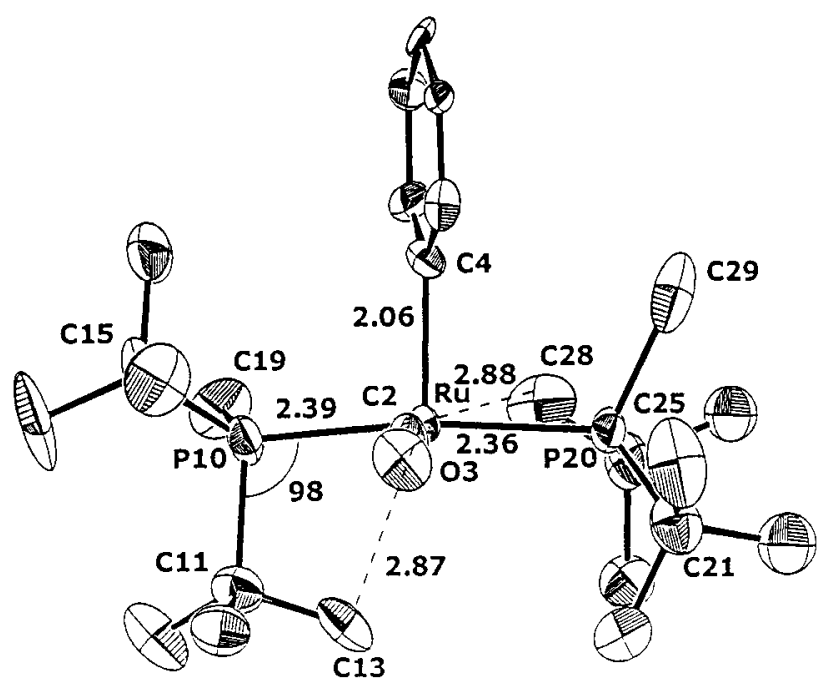

b

Figure 3. ORTEP diagrams of $\mathrm{Ru}(\mathrm{Ph}) \mathrm{Cl}(\mathrm{CO})\left(\mathrm{P}^{\mathrm{t}} \mathrm{Bu}_{2} \mathrm{Me}\right)_{2}, 12$ (a), and $\left[\mathrm{RuPh}(\mathrm{CO})\left(\mathrm{P}^{\mathrm{t} B \mathrm{Bu}_{2} \mathrm{Me}}\right)_{2}\right] \mathrm{BAr}{ }_{4}^{\prime}, \mathbf{1 6}$ (b). Hydrogen atoms are omitted.

\section{Scheme 1}

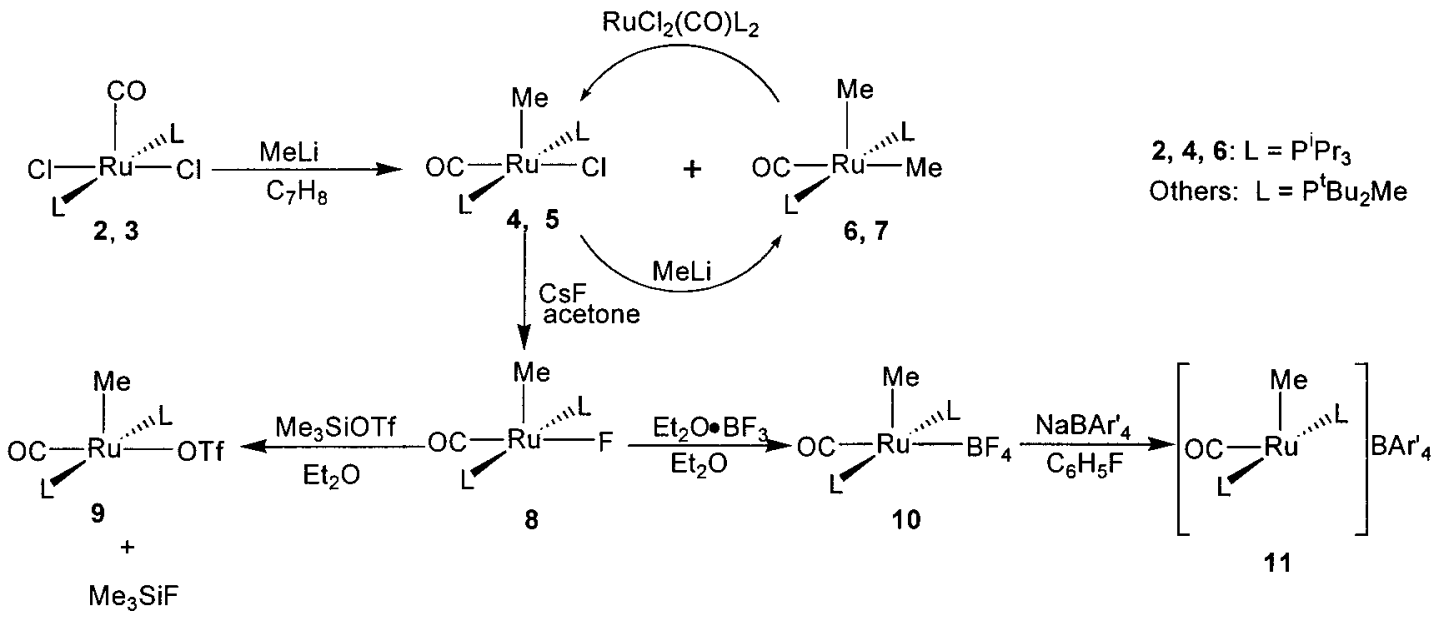

with the weaker donating ability of OTf than of chloride. However, OTf cannot be completely replaced by weakly coordinating $\mathrm{BAr}_{4}{ }_{4}^{-}\left(\mathrm{Ar}^{\prime}=3,5\right.$-trifluoromethyl phenyl). Thus, stirring equimolar 9 and $\mathrm{NaBAr}_{4}{ }_{4}$ in $\mathrm{CH}_{2} \mathrm{Cl}_{2}$ for $12 \mathrm{~h}$ only results in partial replacement of OTf, as evidenced by IR spectroscopy to give a solution having two CO bands (1951 and $1914 \mathrm{~cm}^{-1}$ ) with similar intensity. The higher frequency band is assigned to $\left[\mathrm{RuMe}(\mathrm{CO}) \mathrm{L}_{2}\right] \mathrm{BAr}^{\prime}{ }_{4}, \mathbf{1 1}$, which was synthesized (Scheme 1) from salt metathesis of $\mathrm{NaBAr}_{4}^{\prime}$ and $\mathbf{1 0}\left(\mathrm{C}_{6} \mathrm{H}_{5} \mathrm{~F}, 10 \mathrm{~min}\right) . \mathbf{1 1}$ is a rare example of a 14-electron four-coordinate $\mathrm{Ru}(\mathrm{II})$ alkyl complex. It might be possible to have two agostic interactions as we observed for the similar complex $\left[\mathrm{Ru}(\mathrm{Ph})(\mathrm{CO}) \mathrm{L}_{2}\right] \mathrm{BAr}_{4}{ }_{4}$ (vide infra). Alternatively, the $\mathrm{Ru}-$ methyl group could have an $\alpha$-agostic interaction with the metal, which would cause higher field shift of the methyl proton. However, at $20^{\circ} \mathrm{C}$, this $\mathrm{CH}_{3}$ proton has a normal chemical shift $\left(1.14 \mathrm{ppm}\right.$ in $\mathrm{C}_{6} \mathrm{D}_{5} \mathrm{~F} /$ $\mathrm{C}_{7} \mathrm{D}_{8}$ 1:2). This signal does not change position upon cooling to $-70{ }^{\circ} \mathrm{C}$, and therefore, no $\alpha$-agostic interaction is substantiated. The solvent, $\mathrm{C}_{6} \mathrm{D}_{5} \mathrm{~F}$ and toluene- $d_{8}$, is not likely to be coordinating to the metal, since $\left[\mathrm{RuH}(\mathrm{CO}) \mathrm{L}_{2}\right] \mathrm{BAr}_{4}{ }_{4}$ does not coordinate these solvents. ${ }^{23}$ The two vacant sites of $\mathbf{1 1}$ are likely

(23) Huang, D.; Huffman, J. C.; Bollinger, J. C.; Eisenstein, O.; Caulton, K. G. J. Am. Chem. Soc. 1997, 119, 7398. to be occupied by $\mathrm{C}-\mathrm{H}$ bonds from the ${ }^{t} \mathrm{Bu}$ methyl on the phosphine ligands.

Synthesis and Structure of $\mathbf{R u}(\mathrm{Ph}) \mathrm{Cl}(\mathrm{CO})\left(\mathrm{P}^{t} \mathrm{Bu}_{2} \mathrm{Me}\right)_{2}$. $\mathrm{PhLi}$ reacts with $\mathbf{3}$ at low temperature with clean formation of $\mathrm{RuPhCl}(\mathrm{CO}) \mathrm{L}_{2}, 12$ (eq 3). Excess $\mathrm{PhLi}$, however, does not cause

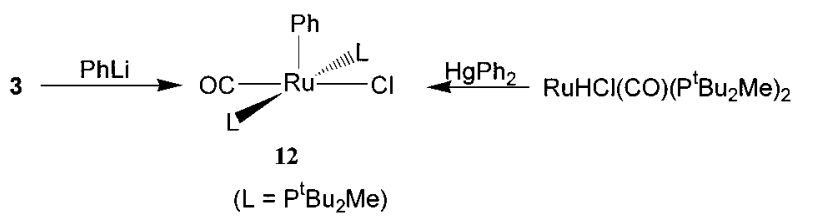

further replacement of the other chloride, probably due to steric crowding in 12. Reaction of $\operatorname{RuH}(\mathrm{Ph})(\mathrm{CO}) \mathrm{L}_{2}$ with excess $N$-chlorosuccinimide (NCS) also gives $\mathbf{1 2}$ in moderate yield. ${ }^{24}$ Alternatively, refluxing $\mathrm{RuHCl}(\mathrm{CO}) \mathrm{L}_{2}$ with $\mathrm{Ph}_{2} \mathrm{Hg}$ in toluene gives $\mathbf{1 2}$ in excellent yield (>80\%). ${ }^{24} \mathbf{1 2}$ is moderately air stable and can be recrystallized from methanol (!). The spectroscopic data of $\mathbf{1 2}$ have been reported and discussed before. ${ }^{23}$ A single crystal of $\mathbf{1 2}$ grown from methanol was chosen for the X-ray study. The ORTEP drawing of $\mathbf{1 2}$ is shown in Figure 3a, and

(24) Roper, W. R.; Wright, L. J. J. Organomet. Chem. 1977, 142, C1. 


\section{Scheme 2}

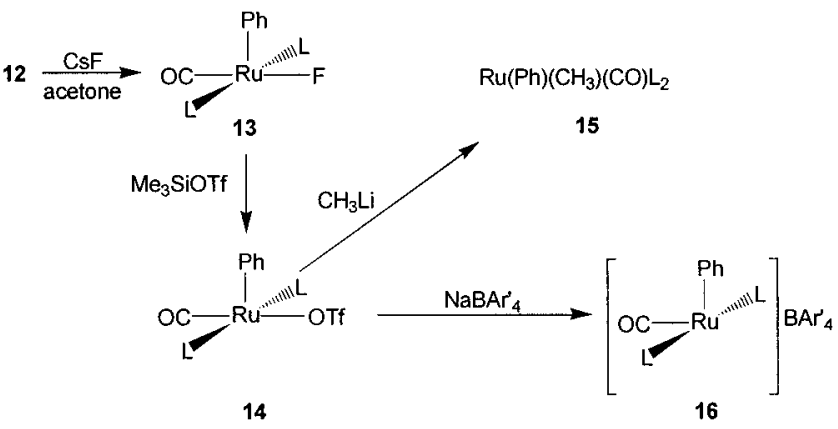

the geometric parameters are in Table 4. Similar to 3 and 4, 12 has a square-based pyramidal geometry but with $\mathrm{Ph}$ trans to the vacant site, consistent with the stronger trans influence of $\mathrm{Ph}$ than $\mathrm{CO}$. Moreover, with $\mathrm{CO}$ trans to $\mathrm{Cl}$, push-pull stabilization is maximized. Although two ${ }^{t} \mathrm{Bu}$ groups on phosphine ligands point toward the vacant site, the shortest distance of the phosphine carbon to $\mathrm{Ru}$ is $3.24 \AA$, and the $\mathrm{Ru}-$ $\mathrm{P}-\mathrm{C}\left(\mathrm{CH}_{3}\right)_{3}$ angles do not deviate much from the normal value of $115^{\circ}$; therefore, there is no agostic interaction. The structure of the related complex $\mathrm{Ru}(p$-tolyl $) \mathrm{Cl}(\mathrm{CO})\left(\mathrm{PPh}_{3}\right)_{2}$ shows weak agostic donations from one ortho phenyl of both phosphines $(\mathrm{Ru} / \mathrm{H}=2.77-2.85 \AA \mathrm{Ru} / \mathrm{C}=3.41 \AA)$ to the site trans to the $p$-tolyl in a square pyramidal structure. ${ }^{25}$ Such weak interactions to $\mathrm{PPh}_{3}$ are absent in $\mathrm{RuCl}(o$-tolyl $)(\mathrm{CO})\left(\mathrm{PPh}_{3}\right)_{2}$, where the $o$-tolyl methyl appears to form an agostic interaction to $\mathrm{Ru}$ $(\mathrm{Ru} / \mathrm{H}=1.9 \AA)$ and $o$-tolyl is no longer in the apical site of a square pyramid. ${ }^{10}$

Halide Replacement of $\mathbf{1 2}$. The $\mathrm{Cl}$ of $\mathbf{1 2}$ can be replaced with $\mathrm{F}$ by salt metathesis with $\mathrm{CsF}$ (acetone, $20{ }^{\circ} \mathrm{C}, 12 \mathrm{~h}$ ) to give $\mathrm{RuPhF}(\mathrm{CO}) \mathrm{L}_{2}, \mathbf{1 3}$ (Scheme 2). 13 is isolated as orange crystals from pentane or by sublimation at $140{ }^{\circ} \mathrm{C}$ at $5 \times 10^{-2}$ mmHg. The ${ }^{31} \mathrm{P}\left\{{ }^{1} \mathrm{H}\right\} \mathrm{NMR}$ of $\mathbf{1 3}$ shows a doublet $\left(J_{\mathrm{PF}}=24\right.$ $\mathrm{Hz}$ ) and, correspondingly, the ${ }^{19} \mathrm{~F}$ spectrum is a triplet close $(-204 \mathrm{ppm})$ to the ${ }^{19} \mathrm{~F}$ chemical shift of $8(-201 \mathrm{ppm})$. There are also five distinct phenyl proton resonances, indicative of slow rotation of $\mathrm{Ph}$ around the $\mathrm{Ru}-\mathrm{C}$ (ipso) bond.

The CO stretching band appears at lower frequency (1890) than that of 12 (1902) since $\mathrm{F}$ is a stronger $\pi$-donor than $\mathrm{Cl}^{26}$ Replacement of $\mathrm{F}$ by triflate occurs under mild conditions using $\mathrm{Me}_{3} \operatorname{SiOTf}\left(\mathrm{Et}_{2} \mathrm{O}, 20{ }^{\circ} \mathrm{C}\right.$, immediate reaction) to give $\mathrm{Ru}(\mathrm{Ph})$ $(\mathrm{OTf})(\mathrm{CO}) \mathrm{L}_{2}, 14$, quantitatively. Ligand exchange of $\mathrm{Me}_{3} \mathrm{Si}-\mathrm{X}$ with metal fluoride has been reported on several occasions. ${ }^{27}$ Surprisingly, salt metathesis using AgOTf does not give the same product; instead, decomposition of $\mathbf{1 2}$ yields [ $\left.\mathrm{AgL}_{2}\right] \mathrm{OTf}$. 14 can also be synthesized in high yield from $\mathrm{RuH}(\mathrm{OTf})(\mathrm{CO})$ $\mathrm{L}_{2}{ }^{23}$ and $\mathrm{Ph}_{2} \mathrm{Hg}$ in refluxing toluene. 14 is soluble in nonpolar solvents such as benzene and pentane, indicative of coordinated triflate. Moreover, similar to $\mathbf{1 2}$ and 13, 14 also has five distinct proton NMR signals for $\mathrm{Ph}$, indicative of slow rotation of $\mathrm{Ph}$. Consistent with weak donation by OTf, the CO stretching frequency $\left(1921 \mathrm{~cm}^{-1}\right)$ of $\mathbf{1 4}$ is higher than those of $\mathbf{1 2}$ and $\mathbf{1 3}$.

$\mathbf{R u}(\mathbf{P h})\left(\mathbf{C H}_{3}\right)(\mathbf{C O}) \mathbf{L}_{2}$. As a better leaving group, OTf of $\mathbf{1 4}$ is readily replaced by $\mathrm{MeLi}$ to give $\mathrm{RuPh}(\mathrm{Me})(\mathrm{CO}) \mathrm{L}_{2}, \mathbf{1 5}$, at

(25) The original structure in ref 10 has been better reinterpreted in a different space group: Marsh, R. E. Acta Crystallogr., Sect. B (Str. Sci.) 1997, 53, 317

(26) (a) Poulton, J. T.; Folting, K.; Streib, W. E.; Caulton, K. G. Inorg. Chem. 1992, 31, 3190. (b) Tilset, M.; Hamon, J. R.; Hamon, P. J. Chem. Soc., Chem. Commun. 1998, 765.

(27) (a) Doherty, N. M.; Crischlow, S. C. J Am. Chem. Soc. 1987, 109 7906. (b) Hoffman, N. W.; Prokopuk, N.; Robbins, M. J.; Jones, C. M.; Doherty, N. M. Inorg. Chem. 1991, 30, 4177. (c) Cooper, A. C.; Huffman, J. C.; Caulton, K. G. Inorg. Chim. Acta 1998, 270, 261.
$20{ }^{\circ} \mathrm{C}$ in benzene within the time of mixing (Scheme 2). In contrast, substitution of $\mathrm{Cl}$ by $\mathrm{Me}$ in $\mathbf{1 2}$ requires a 10-fold excess of $\mathrm{MeLi}$ and prolonged reaction time ( 3 days). ${ }^{28} \mathbf{1 5}$ (like 6) is a rare example of a 16-electron $\mathrm{Ru}(\mathrm{II})$ complex devoid of $\pi$-basic ligands. Similar to $\mathbf{1 4}, \mathbf{1 5}$ shows five distinct phenyl proton chemical shifts, revealing the slow rotation of the $\mathrm{Ph}$ ring on the ${ }^{1} \mathrm{H}$ NMR time scale. The $\mathrm{Ru}-\mathrm{CH}_{3}$ protons appear as a triplet at $1.07 \mathrm{ppm}\left(J_{\mathrm{PH}}=7 \mathrm{~Hz}\right)$. Unlike the high-field ${ }^{13} \mathrm{C}$ chemical shifts of methyl of $4(-11 \mathrm{ppm})$ and one methyl of 6 $(-15 \mathrm{ppm})$, the methyl ${ }^{13} \mathrm{C}$ chemical shift of $\mathbf{1 5}$ is at lower field $(9.5 \mathrm{ppm})$, in agreement with $\mathrm{CH}_{3}$ trans to $\mathrm{CO}$, not the vacant site. Although not trans to a $\pi$-donor ligand, the $\mathrm{CO}$ stretching frequency is low $\left(1883 \mathrm{~cm}^{-1}\right)$, due probably to the strong $\sigma$-donating powers of $\mathrm{CH}_{3}$ and $\mathrm{Ph} .15$ contains three strong trans influencing ligands, $\mathrm{Ph}, \mathrm{Me}$, and $\mathrm{CO}$, and they are all good candidates to occupy the apical site. On the basis of the NMR spectral data, we conclude that $\mathrm{Ph}$ of $\mathbf{1 5}$ lies at the apical site, where it is sterically constrained from rotating easily. $\mathrm{Ru}(\mathrm{H})(\mathrm{Ph})(\mathrm{CO}) \mathrm{L}_{2}$, on the other hand, has $\mathrm{Ph}$ in the basal plane since the hydride resonance is at very high field $(-28 \mathrm{ppm})$ and only three proton chemical shifts for $\mathrm{Ph}$ are observed, indicating fast rotation of the phenyl. ${ }^{23}$ These results, along with the geometry of the methyl complexes, permit us to conclude that the trans influence of the $\sigma$-donor ligands has the order of $\mathrm{H}>\mathrm{Ph}>\mathrm{Me}>\mathrm{CO}$.

16-electron, five-coordinate $\mathrm{Ru}(\mathrm{II})$ complexes without $\pi$-donor ligands are rare. So far, only one such compound, RuH$\left(\mathrm{SiHPh}_{2}\right)(\mathrm{CO}) \mathrm{L}_{2}$, was structurally characterized. ${ }^{29}$ Similar complexes $\mathrm{Ru}(\mathrm{H})_{2}(\mathrm{CO}) \mathrm{L}_{2}$ and $\mathrm{RuH}(\mathrm{Ph})(\mathrm{CO}) \mathrm{L}_{2}$ are not long-lived species since they tend to eliminate $\mathrm{H}_{2}$ or benzene. In contrast, 15 remains unchanged in toluene for 2 days at $20^{\circ} \mathrm{C}$. Generally, since metal carbon bonds are weaker than metal hydrogen bonds, the persistence of $\mathbf{1 5}$ should be attributed to the kinetic barrier for reductive elimination to form a $\mathrm{C}-\mathrm{C}$ bond.

Synthesis and Structure of $\left[\operatorname{RuPh}(\mathrm{CO}) \mathrm{L}_{2}\right] \mathrm{BAr}^{\prime}{ }_{4}$. Triflate can be removed from 14 using $\mathrm{NaBAr}_{4}$ to give $[\mathrm{RuPh}(\mathrm{CO})$ $\left.\mathrm{L}_{2}\right] \mathrm{BAr}_{4}{ }_{4}, \mathbf{1 6}$, in either methylene chloride or in fluorobenzene at room temperature in the time of mixing (Scheme 2). The highly air-sensitive complex $\mathbf{1 6}$ is purified by recrystallization as orange crystals from a pentane/fluorobenzene mixture with strict exclusion of air and moisture. At room temperature, the NMR of the Ph protons show only three peaks, including one sharp triplet for the para proton and one broad peak for the meta and one broad peak for the ortho protons. Therefore, the $\mathrm{Ph}$ rotation is faster as compared to $\mathbf{1 2}$ with $\mathrm{Ph}$ at the apical site. Upon cooling to $-70{ }^{\circ} \mathrm{C}$, each broad peak decoalesces to two multiplets. The phosphine peak remains a sharp singlet in the same temperature range; therefore, slow rotation around the $\mathrm{Ru}-\mathrm{P}$ bond is not observed, which might have given rise to two magnetically different phosphines, as is seen for $\mathbf{1 2} .^{30}$ Two virtual triplets for ${ }^{t} \mathrm{Bu}$ groups reveal the nonplanar arrangement of the four ligands. The CO stretching frequency is high (1958 $\mathrm{cm}^{-1}$ ), and two bands with medium strength are also found at the agostic $\mathrm{C}-\mathrm{H}$ stretch region $\left(2722\right.$ and $\left.2672 \mathrm{~cm}^{-1}\right)$. These two bands disappear after $\mathbf{1 6}$ is saturated with excess $\mathrm{CO}$, which replaces the agostic interacting $\mathrm{C}-\mathrm{H}$ bonds (Figure 4 ). These agostic interactions are highly fluxional and cannot be frozen out on the NMR time scale as in the other agostic interactions between unsaturated metal and the phosphine ligand $\mathrm{C}-\mathrm{H}$ bond. ${ }^{31}$ To gain solid evidence for the structure of $\mathbf{1 6}$, an X-ray

(28) Ogasawara, M. Personal communication.

(29) Heyn, R. H.; Huffman, J. C.; Caulton, K. G. New J. Chem. 1993, $17,797$.

(30) Notheis, J. U.; Heyn, R. H.; Caulton, K. G. Inorg. Chim. Acta 1995, 229, 187. 


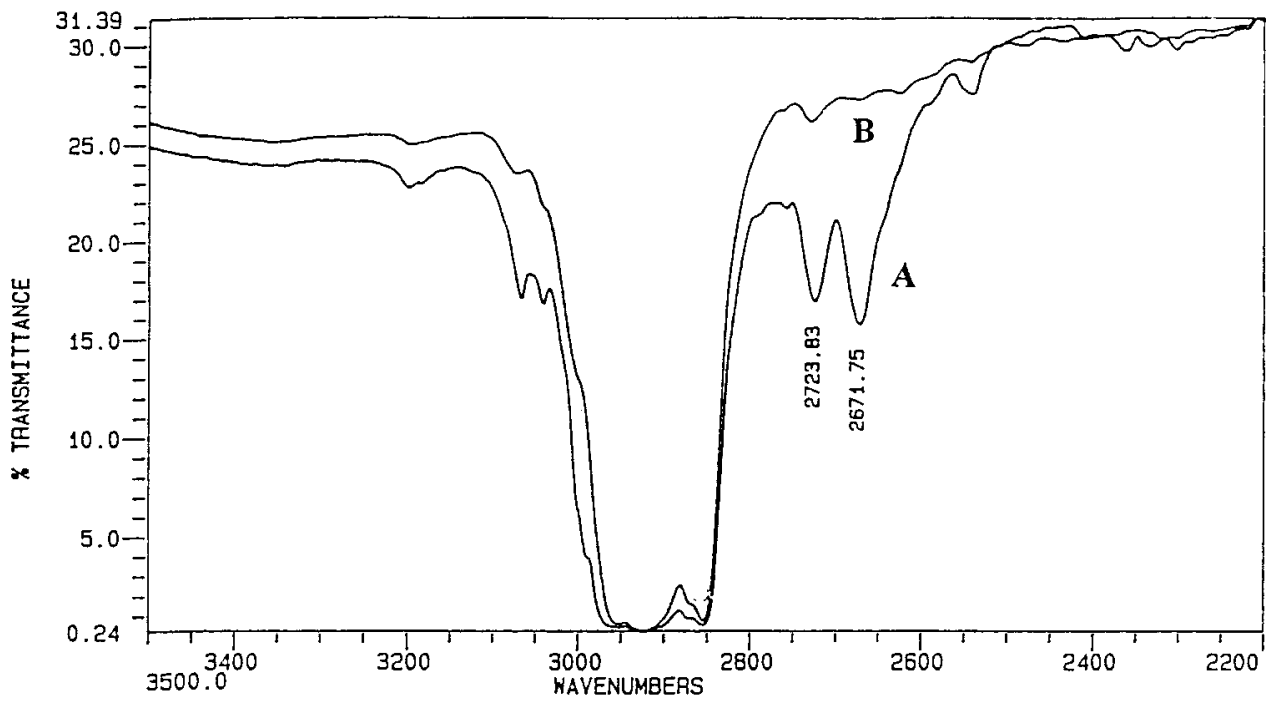

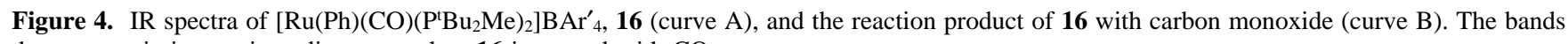
due to agostic interactions disappear when $\mathbf{1 6}$ is treated with $\mathrm{CO}$.

crystal structure study was carried out. The ORTEP diagram is shown in Figure $3 \mathrm{~b}$ and the geometric parameters are in Table 5. Four-coordinate $\mathbf{1 6}$ adopts a sawhorse geometry with two phosphine ligands trans and the $\mathrm{Ph}$ and $\mathrm{CO}$ cis to each other. The two vacant sites are occupied by agostic $\mathrm{C}-\mathrm{H}$ bonds from two ${ }^{t} \mathrm{Bu}$ on different phosphines. The $\mathrm{Ru} / \mathrm{C}_{\text {agostic }}$ distances are short (2.88 and $2.87 \AA$, respectively), indicative of relatively strong interactions. Comparing the structural differences of $\mathbf{1 6}$ and 12 (Figure 3a,b) provides some insight into the impact of the structural changes upon removal of the $\mathrm{X}$ ligand. Removal of the $\mathrm{X}$ ligand does not cause any large disturbance to the remaining four atoms bound to $\mathrm{Ru}$; they remain approximately in the same relative position. The $\mathrm{P}-\mathrm{Ru}-\mathrm{P}$ angles and $\mathrm{CO}-\mathrm{Ru}-\mathrm{C}$ angles are comparable between $\mathbf{1 6}$ and 12. The $\mathrm{Ru}-\mathrm{P}$ distances are shorter in 16. In sharp contrast to small movements of atoms directly bound to $\mathrm{Ru}$, the substituents on phosphine have been disturbed significantly. The angle $\mathrm{Ru}-\mathrm{P} 3-\mathrm{C} 5$ of $\mathbf{1 2}$ is $108^{\circ}$, while it is $10^{\circ}$ smaller in 16. In 12, the angle $\mathrm{Ru}-\mathrm{P} 13-\mathrm{C} 19$ is $119^{\circ}$; in contrast, upon removal of the $\mathrm{Cl}$, this angle decreases to $97^{\circ}$ $(\mathrm{Ru}-\mathrm{P} 20-\mathrm{C} 25)$. Accordingly, the $\mathrm{Ru} / \mathrm{C}_{\text {agostic }}$ distances are shortened by 0.375 and $0.874 \AA$, respectively, compared to the corresponding $\mathrm{Ru} / \mathrm{C}$ distance in 12. Thus, the removal of the $\mathrm{Cl}$ creates two agostic interactions. The absence of agostic interaction in $\mathbf{1 2}$ and the presence of two agostic interactions in $\mathbf{1 6}$ show that agostic interaction is not solely determined by the nature of the ligand trans to the empty site. If that were the case, one should have observed an agostic interaction trans to $\mathrm{Ph}$ in 12. Going from a $16 \mathrm{e} \mathrm{Ru}(\mathrm{Ph}) \mathrm{Cl}(\mathrm{CO}) \mathrm{L}_{2}$ to $14 \mathrm{e} \mathrm{RuPh}-$ (CO) $\mathrm{L}_{2}{ }^{+}$is likely to lower all empty metal orbitals, even those which in first approximation should not have been influenced by the presence of the removed ligand $\left(\mathrm{Cl}^{-}\right)$; there is a general increase in electrophilicity.

Reactivity of 16. Surprisingly, this highly electrophilic complex is thermally robust in solvents such as benzene or toluene. Upon heating in toluene- $d_{8}\left(100{ }^{\circ} \mathrm{C}\right)$ for $24 \mathrm{~h}$, no significant decomposition or reaction is evidenced by NMR spectroscopy. The ${ }^{1} \mathrm{H}$ NMR spectrum remains unchanged. Moreover, the two diastereotopic ${ }^{t} \mathrm{Bu}$ groups do not decoalesce at $100{ }^{\circ} \mathrm{C}$ (toluene- $d_{8}$ ), thus there is no phosphine dissociation or unimolecular inversion through a square-planar intermediate.

(31) Heinekey, D. M.; Radzewich, C. E.; Voges, M. H.; Schember, B. M. J. Am. Chem. Soc. 1997, 119, 4172.
On the other hand, it shows reactivity of the $\mathrm{Ru}-\mathrm{Ph}$ with $\mathrm{E}-\mathrm{H}$ bonds ( $E=$ boryl, $\mathrm{H}$, and $\mathrm{C}(\mathrm{sp})$ ).

(A) With Catecholborane, Synthesis of $\left[\mathrm{RuB}\left(\mathrm{C}_{6} \mathrm{H}_{4} \mathrm{O}_{2}\right)\right.$ $\left.(\mathbf{C O}) \mathbf{L}_{2}\right] \mathbf{B A r}^{\prime}{ }_{4}$. A mixture of 1 equiv catecholborane and $\mathbf{1 6}$ in methylene chloride produces exclusively $\left[\mathrm{RuH}(\mathrm{CO}) \mathrm{L}_{2}\right] \mathrm{BAr}_{4}{ }_{4}$ and $\left(\mathrm{C}_{6} \mathrm{H}_{4} \mathrm{O}_{2}\right) \mathrm{B}-\mathrm{Ph}$ in $1 \mathrm{~h}$ at room temperature (eq 4). The

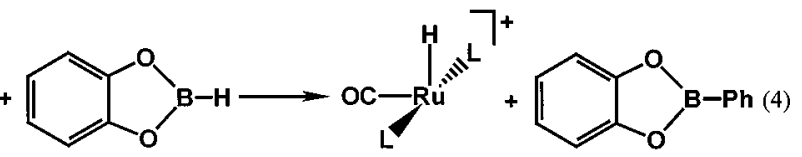

products are identified by comparing the ${ }^{1} \mathrm{H}$ and ${ }^{31} \mathrm{P}$ NMR spectra with the known values for the two compounds. 14 also reacts (Scheme 3 ) with 1 equiv catecholborane, albeit at higher temperature $\left(80{ }^{\circ} \mathrm{C}, 4 \mathrm{~h}\right)$, to give $\mathrm{RuH}(\mathrm{OTf})(\mathrm{CO}) \mathrm{L}_{2}(90 \%)$, trace $\mathrm{Ru}\left(\mathrm{BR}_{2}\right)(\mathrm{OTf})(\mathrm{CO}) \mathrm{L}_{2}, \mathbf{1 7}$, and $\left(\mathrm{C}_{6} \mathrm{H}_{4} \mathrm{O}_{2}\right) \mathrm{B}-\mathrm{Ph}$. If one more equivalent of catecholborane is added to the reaction mixture, clean conversion to $\mathbf{1 7}$ is achieved. Therefore, the formation of 17 from 14 involves two steps to release $\left(\mathrm{C}_{6} \mathrm{H}_{4} \mathrm{O}_{2}\right) \mathrm{B}-\mathrm{Ph}$ and $\mathrm{H}_{2}$ separately (Scheme 3). Indeed, reaction of $\mathrm{RuH}(\mathrm{OTf})(\mathrm{CO})-$ $\mathrm{L}_{2}$ with 1 equiv catecholborane cleanly yields $\mathbf{1 7}$. 17 has two ${ }^{t} \mathrm{Bu}$ virtual triplets and a broad singlet for ${ }^{31} \mathrm{P}$ and ${ }^{11} \mathrm{~B}(44.5$ ppm) signals. The $\mathrm{CO}$ stretching band appears at higher frequency $\left(1939 \mathrm{~cm}^{-1}\right)$ than that of $\mathbf{6}$, in agreement with the presence of the $\pi$-acidic boryl ligand. The mechanism of this reaction can be oxidative addition followed by reductive elimination or $\sigma$-bond metathesis. In either mechanism, the reaction is highly selective for the formation of $\mathrm{M}-\mathrm{BR}_{2}$. Hartwig and co-workers studied the mechanism of the reaction of saturated $\mathrm{CpRu}\left(\mathrm{PPh}_{3}\right)_{2} \mathrm{Me}$ and catecholborane (giving metal hydride and methylcatecholborane) and concluded that the reaction proceeds by a four-centered transition state ( $\sigma$-bond metathesis), not by oxidative addition. ${ }^{32}$ Although a similar mechanism may be operative in eq 4 , since the $\mathrm{Ru}$ of $\mathbf{1 6}$ is already $\pi$-electron deficient and oxidative addition to give $\mathrm{aRu}$ (IV) species is not favored, highly unsaturated $\mathbf{1 6}$ is likely to coordinate catecholborane before further reaction occurs. Recently, Roper and co-workers reported that the reaction of either $\mathrm{RuHCl}(\mathrm{CO})\left(\mathrm{PPh}_{3}\right)_{3}$ or $\mathrm{Ru}(\mathrm{Ph}) \mathrm{Cl}(\mathrm{CO})\left(\mathrm{PPh}_{3}\right)_{2}$ with catecholborane gives $\mathrm{Ru}$ (boryl) $\mathrm{Cl}(\mathrm{CO})\left(\mathrm{PPh}_{3}\right)_{2}$ as the sole product. How-

(32) Hartwig, J. H.; Bhandari, S.; Rablen, P. R. J. Am. Chem. Soc. 1994 116, 1839. 
Scheme 3

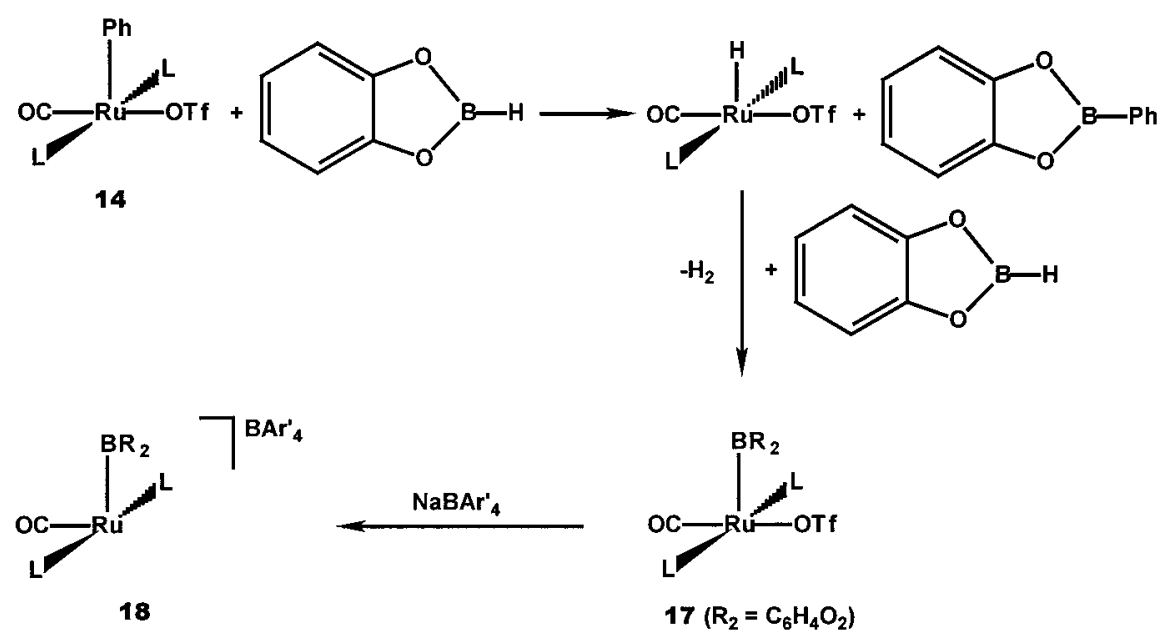

ever, no experimental details concerning the reaction of $\mathrm{Ru}(\mathrm{Ph}) \mathrm{Cl}(\mathrm{CO})\left(\mathrm{PPh}_{3}\right)_{2}$ are given. Therefore, it is not clear if the reaction is also a two-step process, with initial formation of $\mathrm{RuHCl}(\mathrm{CO})\left(\mathrm{PPh}_{3}\right)_{2}$, which then further reacts with catecholborane. In contrast, the Os analogue $\mathrm{OsHCl}(\mathrm{CO})\left(\mathrm{PPh}_{3}\right)_{3}$ does not react with catecholborane, while $\mathrm{Os}(\mathrm{Ph}) \mathrm{Cl}(\mathrm{CO})\left(\mathrm{PPh}_{3}\right)_{2}$ does, to give the boryl complex $\mathrm{Os}\left(\mathrm{BR}_{2}\right) \mathrm{Cl}(\mathrm{CO})\left(\mathrm{PPh}_{3}\right)_{2}$ and benzene. ${ }^{33}$ Saturated $\mathrm{OsHCl}(\mathrm{CO})\left(\mathrm{PPh}_{3}\right)_{3}$ may not undergo a ligand dissociation under the reaction conditions, which is a prerequisite for the reaction to occur, while $\mathrm{RuHCl}(\mathrm{CO})\left(\mathrm{PPh}_{3}\right)_{3}$ may have a labile phosphine.

$\left[\mathbf{R u B}\left(\mathbf{O}_{2} \mathbf{C}_{6} \mathbf{H}_{4}\right)(\mathbf{C O}) \mathbf{L}_{2}\right] B \mathbf{A r}^{\prime}{ }_{4}$. Salt metathesis of $\mathbf{1 7}$ with $\mathrm{NaBAr}_{4}{ }_{4}$ in methylene chloride or fluorobenzene gives [Ru$\left.\left(\mathrm{BR}_{2}\right)(\mathrm{CO}) \mathrm{L}_{2}\right] \mathrm{BAr}^{\prime}{ }_{4}, \mathbf{1 8}$, in quantitative yield (Scheme 3$)$. Like other four-coordinated $\mathrm{RuR}(\mathrm{CO}) \mathrm{L}_{2}{ }^{+}, \mathbf{1 8}$ exhibits two virtual triplets for the ${ }^{t} \mathrm{Bu}$ groups indicative of nonplanar geometry with two phosphines mutually trans. Only two proton chemical shifts are observed for catecholboryl, indicating fast rotation of the boryl group. ${ }^{11} \mathrm{~B} \mathrm{NMR}$ and ${ }^{31} \mathrm{P}\left\{{ }^{1} \mathrm{H}\right\}$ NMR spectra are broad singlets probably caused by the quadrupolar ${ }^{11} \mathrm{~B}$. The $\mathrm{CO}$ stretching band of $\mathbf{1 8}\left(1981 \mathrm{~cm}^{-1}\right)$ is higher than any other $\left[\mathrm{RuR}(\mathrm{CO}) \mathrm{L}_{2}\right] \mathrm{BAr}_{4}^{\prime}$ owing to the $\pi$-acidic boryl group. To our knowledge, 18 is the first example of a highly electron-deficient (14e) $\mathrm{Ru}(\mathrm{II})$ boryl complex, the reactivity of which is still under investigation and will be reported separately.

(B) Reaction with $\mathrm{Me}_{3} \mathrm{SiCCH}$. Addition of 1 equiv of $\mathrm{Me}_{3}-$ $\mathrm{SiCCH}$ to a methylene chloride solution of $\mathbf{1 6}$ at room temperature gives in the time of mixing partial conversion to $\mathrm{PhCCSiMe}_{3},\left[\mathrm{Ru}\left\{\eta^{3}-\left(\mathrm{Me}_{3} \mathrm{SiCH}=\mathrm{C}-\mathrm{CH}=\mathrm{CH}\left(\mathrm{SiMe}_{3}\right)\right\}(\mathrm{CO}) \mathrm{L}_{2}\right]-\right.$ $\mathrm{BAr}_{4}^{\prime}, 19$, and a trace amount of $\left[\mathrm{Ru}\left(\mathrm{CH}=\mathrm{CH}\left(\mathrm{SiMe}_{3}\right)(\mathrm{CO}) \mathrm{L}_{2}\right]-\right.$ $\mathrm{BAr}_{4}{ }_{4}, 20$ (Scheme 4). If two more equivalents of $\mathrm{Me}_{3} \mathrm{SiCCH}$ are added, clean conversion to $\mathbf{1 9}$ is observed. 19 can be synthesized independently from $\mathrm{RuH}(\mathrm{CO}) \mathrm{L}_{2}{ }^{+}$with 2 equiv of $\mathrm{Me}_{3} \mathrm{SiCCH}$, and its structure has been determined. ${ }^{11}$ Therefore, is it likely that the reaction of $\mathbf{1 6}$ with $\mathrm{Me}_{3} \mathrm{SiCCH}$ forms $\mathrm{RuH}-$ (CO) $\mathrm{L}_{2}^{+}$, which further reacts with $\mathrm{Me}_{3} \mathrm{SiCCH}$ to give 19. The most straightforward mechanism of the first step is that $\mathrm{Me}_{3}-$ $\mathrm{SiCCH}$ and 16 undergo $\sigma$-bond metathesis to give $\mathrm{Me}_{3} \mathrm{SiCCPh}$ and $\mathrm{RuH}(\mathrm{CO}) \mathrm{L}_{2}{ }^{+}$, which then reacts with $\mathrm{Me}_{3} \mathrm{SiCCH}$ to give 19 and 20. Alternatively, oxidative addition of the $\mathrm{C}(\mathrm{sp})-\mathrm{H}$ bond to $\mathrm{Ru}(\mathrm{II})$ followed by exclusive reductive elimination of $\mathrm{Me}_{3} \mathrm{SiCCPh}$ would also account for the first step. To gain more information on this reaction, a low-temperature NMR spectroscopic study was carried out. One equivalent of $\mathrm{Me}_{3} \mathrm{SiCCH}$ and

(33) Irvine, G. J.; Roper, W. R.; Wright, L. J. Organometallics 1997, $16,2291$.

16 were mixed at $-70{ }^{\circ} \mathrm{C}$ in an $\mathrm{NMR}$ tube in $\mathrm{CD}_{2} \mathrm{Cl}_{2}$. At temperatures below $-40{ }^{\circ} \mathrm{C}$, there is no detectable interaction between 16 and $\mathrm{Me}_{3} \mathrm{SiCCH}$. As the temperature rises, one new product starts to form, which has a characteristic vinyl proton triplet $\left(J_{\mathrm{PH}}=2 \mathrm{~Hz}\right)$ at $5.87 \mathrm{ppm}$. At $-5{ }^{\circ} \mathrm{C}$, this product is the dominant one ( $>70 \%$, based on ${ }^{31} \mathrm{P}$ NMR integration). ${ }^{31} \mathrm{P} \mathrm{NMR}$ of this product is a sharp singlet and two virtual ${ }^{t} \mathrm{Bu}$ triplets $\left({ }^{1} \mathrm{H}\right.$ NMR) are also identified in addition to a singlet for $\mathrm{Me}_{3} \mathrm{Si}$. Therefore, it has two trans phosphines with diastereotopic ${ }^{t} \mathrm{Bu}$ groups. On the basis of these data, we propose that the product has structure 21. The formation of $\mathbf{2 1}$ requires that one $\mathrm{Me}_{3} \mathrm{SiCCH}$ isomerized to vinylidene before the $\mathrm{Ph}$ migratory insertion occurs. Further warming in the presence of free $\mathrm{Me}_{3} \mathrm{SiCCH}$ converts some 21 to $\mathrm{PhCCSiMe}_{3}, \mathbf{1 9}$ and 20 until all $\mathrm{Me}_{3} \mathrm{SiCCH}$ is consumed (judging from ${ }^{1} \mathrm{H}$ NMR). 21 then isomerizes at room temperature to $\mathbf{2 2}$, which has been synthesized independently from $\mathrm{RuH}(\mathrm{CO}) \mathrm{L}_{2}{ }^{+}$and $\mathrm{PhCCSiMe}_{3}$ and characterized by X-ray diffraction. ${ }^{34}$ The transformation of $\mathbf{2 1}$ to $\mathbf{2 2}$ is likely to go through $\beta$-hydrogen migration via an unobserved intermediate, the $\eta^{2}-\mathrm{Ph}-\mathrm{CC}-\mathrm{SiMe}_{3}$ adduct 23 . The final reaction mixture gives over $80 \% \mathbf{2 2}$, and small amounts of 19 and 20. Although 22 might have been formed via direct addition of $\mathrm{Ru}-\mathrm{Ph}$ to the $\mathrm{C} \equiv \mathrm{C}$ bond, this cannot account for the complexity of the observed intermediates. Consistently, if the same reaction is carried out using 3 equiv $\mathrm{Me}_{3} \mathrm{SiCCH}$, at low temperature $\left(<-5^{\circ} \mathrm{C}\right), \mathbf{2 1}$ is the dominant product, which releases $\mathrm{PhCCSiMe}_{3}$ and transforms to 19. No 22 is observed. The high migrating ability of the silyl, hydrogen, and phenyl groups makes this reaction complicated and likely leads to the thermodynamic product.

\section{Conclusion}

We have demonstrated the synthesis and structural characterization of the 16-electron five-coordinate $\mathrm{Ru}(\mathrm{II})$ complexes and 14-electron four-coordinated $\mathrm{Ru}(\mathrm{Ph})(\mathrm{CO}) \mathrm{L}_{2}{ }^{+}$, and their reactivity toward $\mathrm{E}-\mathrm{H}$ bonds is also examined. On the basis of the results gathered here, several conclusions can be reached.

(1) Synthesis of the 14-electron four-coordinate complex, $\left[\mathrm{RuR}(\mathrm{CO}) \mathrm{L}_{2}\right] \mathrm{BAr}^{\prime}{ }_{4}$, is achieved by salt metathesis of its triflate precursor and $\mathrm{NaBAr}_{4}{ }_{4}$. The cation adopts a sawhorse geometry with two sterically demanding phosphine ligands trans and the two strong trans influencing ligands cis to each other so that the unsaturated metal gains the most steric protection. This also

(34) Huang, D.; Streib, W. E.; Eisenstein, O.; Caulton, K. G. J. Am. Chem. Soc., in press. 


\section{Scheme 4}

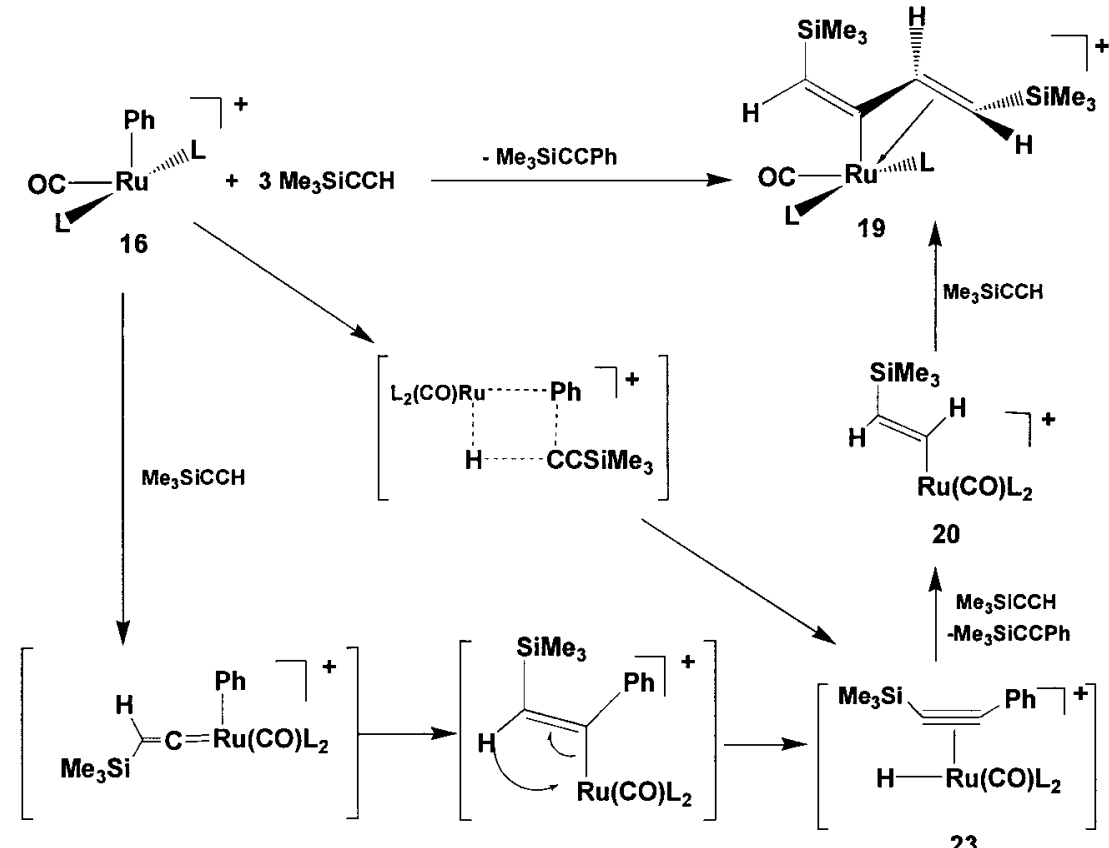

21

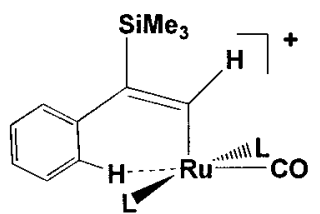

22

raises the energy of the empty valence orbitals. The two vacant sites are occupied by agostic interactions, which is directly proved, in the case of $\mathrm{R}=\mathrm{Ph}$, by $\mathrm{X}$-ray single-crystal structure analysis and the solid-state IR spectrum. Structural comparison of $\mathrm{Ru}(\mathrm{Ph})(\mathrm{CO}) \mathrm{L}_{2}{ }^{+}$with its five-coordinated precursor $\mathrm{Ru}(\mathrm{Ph})$ $\mathrm{Cl}(\mathrm{CO}) \mathrm{L}_{2}$ reveals that halide removal does not cause a major geometry change of the remaining fragment; on the other hand, two agostic interactions are created.

(2) 16-electron $\mathrm{Ru}(\mathrm{II})$ carbyl complexes $\mathrm{RuR}_{2}(\mathrm{CO}) \mathrm{L}_{2}$ without $\pi$-donor ligands are persistent species. This is in sharp contrast to the hydride complexes, $\mathrm{Ru}(\mathrm{H})(\mathrm{Ph})(\mathrm{CO}) \mathrm{L}_{2}$ and $\mathrm{RuH}-$ $(\mathrm{Me})(\mathrm{CO}) \mathrm{L}_{2}$, which readily undergo reductive elimination even at $-40^{\circ} \mathrm{C}\left(\mathrm{RuH}(\mathrm{Me})(\mathrm{CO}) \mathrm{L}_{2}\right)$. The persistence of the carbyl complexes can be attributed to kinetic sluggishness of reductive elimination.

(3) On the basis of the geometry preference of five-coordinate complexes, the magnitude of trans influence has the following order: $\mathrm{H}>\mathrm{Ph}>\mathrm{CH}_{3}>\mathrm{CO}>\mathrm{Cl}$.
The sawhorse geometry of $\mathrm{Ru}(\mathrm{R})(\mathrm{CO}) \mathrm{L}_{2}{ }^{+}$places a vacant site cis to the $\mathrm{M}-\mathrm{R}$ bond. In combination with the Lewis acidity of the metal (as demonstrated by the two agostic interactions), we can envision some interesting reactivity between an incoming ligand and $\mathrm{M}-\mathrm{R}$. The detailed reactivity study will be pursued and reported in due course.

Acknowledgment. This work is supported by the National Science Foundation. We also thank the Deutsche Forschungsgemeinschaft and Prof. Dr. W. Kaim for financial support and Johnson Matthey/Aesar for material support.

Supporting Information Available: Full crystallographic details, positional and thermal parameters, and distances and angles on four compounds (PDF). This material is available free of charge via the Internet at http://pubs.acs.org.

JA990621W 